\title{
Le journal de Mademoiselle Anne-Pauline Gilbert (1926-1927) : Genre, éducation protestante et colonisation à Tahiti pendant l'entre-deux-guerres
}

\author{
Gwendoline Malogne-Fer \\ Groupe Sociétés, Religions, Laïcités (CNRS-EPHE), Paris, France
}

\begin{abstract}
Résumé
Mademoiselle Anne-Pauline Gilbert part à Tahiti en 1926 envoyée par la Société des missions évangéliques de Paris comme institutrice bénévole. Elle tient un journal, intitulé «Récit de voyage et de premières impressions d'une institutrice missionnaire à Tahiti » pendant les dix-huit premiers mois, qui est à mi-chemin entre le journal intime, le récit de voyage et le compte rendu missionnaire. La lecture de ce journal permet d'une part de mieux comprendre les motivations des femmes missionnaires occidentales. Il montre d'autre part la prégnance des stéréotypes culturels et sexués, la volonté et la difficulté de les dépasser ou de s'en accommoder. La position marginale et le regard décalé d'Anne-Pauline Gilbert permettent enfin de mieux comprendre l'imbrication des hiérarchies de sexe et de «race» constitutives des dynamiques missionnaires pendant l'entre-deux-guerres.
\end{abstract}

\begin{abstract}
Miss Anne-Pauline Gilbert arrived in Tahiti in 1926, as a volunteer teacher sent by the Paris Evangelical Missionary Society. During her first 18 months, she kept a diary entitled "An account of the journey and first impressions of a missionary teacher in Tahiti" which is halfway between a travel book and a missionary report. The reading of this diary helps a better understanding of the motivations of Western missionary women. It also shows the influence of cultural and gender stereotypes, a willingness to overcome them, or to cope with them, and adapt to them. The marginal position of Anne-Pauline Gilbert and her misaligned point of view throw light on the intertwining of gender and "race" hierarchies in missionary dynamics during the inter-war years.
\end{abstract}


Mots-clés

Tahiti, Polynésie, Protestantisme, Société des missions évangéliques de Paris, femmes missionnaires, éducation, entre-deux-guerres

Keywords

Tahiti, Polynesia, Protestantism, Paris Evangelical Missionary Society, women missionaries, education, interwar years

Alors que les hommes envoyés par la société des missions évangéliques de Paris (SMEP) dans les îles de Tahiti à partir de 1863 sont pasteurs et mariés, les femmes «missionnaires » sont célibataires et diplômées : Il s'agit d'institutrices envoyées en Polynésie à partir de 1885 et d'infirmières qui exercent à la léproserie d'Orofara à partir de $1924^{1}$. Si les « champs de mission» ont donné l'opportunité à des femmes occidentales d'exercer des responsabilités importantes, l'analyse historique des missions protestantes à Tahiti et dans les «îles adjacentes » reste fondée essentiellement sur des écrits masculins - mémoires, comptes rendus adressés à la direction de la SMEP, journal de la Société etc.

Dans ce contexte de relative rareté des écrits de femmes missionnaires, le journal d'Anne-Pauline Gilbert², envoyée par la SMEP comme institutrice à l'école des jeunes filles de Papeete en 1926, fait exception.

La valorisation des archives personnelles et des sources autobiographiques, depuis une quarantaine d'années au sein de l'historiographie

1) Cet article est issu d'une communication donnée le $1^{\mathrm{er}}$ juin 2011 lors d'une journée d'étude sur les collections de la société des missions évangéliques de Paris au Défap-service protestant de Mission. Je remercie Emilie Gangnat et Claire-Lise Lombard, les organisatrices de cette journée d'étude ainsi que Yannick Fer et Florence Rochefort pour la relecture critique d'une première version de cet article. Pour une analyse des rôles des femmes protestantes, épouses de missionnaires ou célibataires, et de l'évolution de leur engagement au cours des $\mathrm{XIX}^{\mathrm{e}}$ et $\mathrm{XX}^{\mathrm{e}}$ siècles: Deborah Kirkwood, "Protestant Missionary Women: Wives and Spinters» in Fiona Bowie, Deborah Kirkwood \& Shirley Ardener (eds.), Women and Missions: Past and Present. Anthropological and Historical Perceptions, Providence/Oxford, Berg, 1993, pp. 23-42. Pour une étude du rôle des missions protestantes et catholiques dans la fondation des familles chrétiennes et dans l'apprentissage de la domesticité en Océanie : Margaret Jolly \& Martha Macintyre, Family \& Gender in the Pacific. Domestic contradictions and the colonial impact, Cambridge University Press, 1989.

2) Anne-Pauline Gilbert, Récit de voyage et de premières impressions d'une institutrice missionnaire à Tahiti, mars 1926- août 1927, 156 pages. 
française, peut se comprendre comme un moyen de redonner la parole à ceux qui en ont été dépossédés et de constituer des contre-sources à l'histoire officielle ${ }^{3}$. Mais l'usage des archives de soi est aussi lié, de façon plus générale, à la reconnaissance de l'individu ordinaire, de ses émotions et de ses expériences, comme acteur à part entière de l'histoire ${ }^{4}$. Rebecca Rogers souligne ainsi à propos des missions que :

La plus grande ouverture des archives des congrégations religieuses donne incontestablement la possibilité de proposer de nouvelles lectures des rapports entre genre, mission et colonisation en France. Il est temps de montrer qu'au sein du mouvement missionnaire catholique (et peut-être aussi protestant), les femmes ont été des actrices importantes dans la rencontre coloniale [...] La prise en compte de leur histoire permet non seulement de montrer la complexité de l'histoire coloniale, mais aussi de saisir comment les dynamiques de genre la traversent, la structurent et l'orientent ${ }^{5}$.

Le journal d'Anne-Pauline Gilbert est à mi-chemin entre le journal intime, le récit de voyage et le compte rendu missionnaire; il est destiné à être lu mais exclusivement par «quelques amies». La lecture de ce journal permet, d'une part, de mieux comprendre les motivations et les attentes de ces femmes occidentales missionnaires, les représentations sociales et l'imaginaire associé à Tahiti. D’autre part, elle montre la prégnance des stéréotypes culturels et sexués, la volonté et la difficulté de les dépasser ou de s'en accommoder; enfin, la lecture de ce journal offre un regard introspectif et parfois décalé sur l'œuvre missionnaire protestante en Polynésie.

\section{La mission protestante à Tahiti pendant l'entre deux guerres}

L'implantation de la SMEP à Tahiti et dans les îles adjacentes à partir de 1863 est moins liée à des motifs religieux - les missionnaires protestants anglais de la London Missionary Society (LMS) sont présents à Tahiti

\footnotetext{
3) Philippe Artières et Dominique Kalifa, «L'historien et les archives personnelles: Pas à pas », Sociétés et Représentations, 2002, numéro 13, pp. 7-15.

4) Ibidem.

5) Rebecca Rogers, «Genre, mission et colonisation», in Dominique Borne et Benoît Falaize (eds.), Religions et colonisation: Afrique-Asie-Océanie-Amériques, $X V \mathrm{I}^{\mathrm{e}}-X X^{\mathrm{e}}$ siècle. Paris, Editions de l'Atelier, 2009, p. 100.
} 


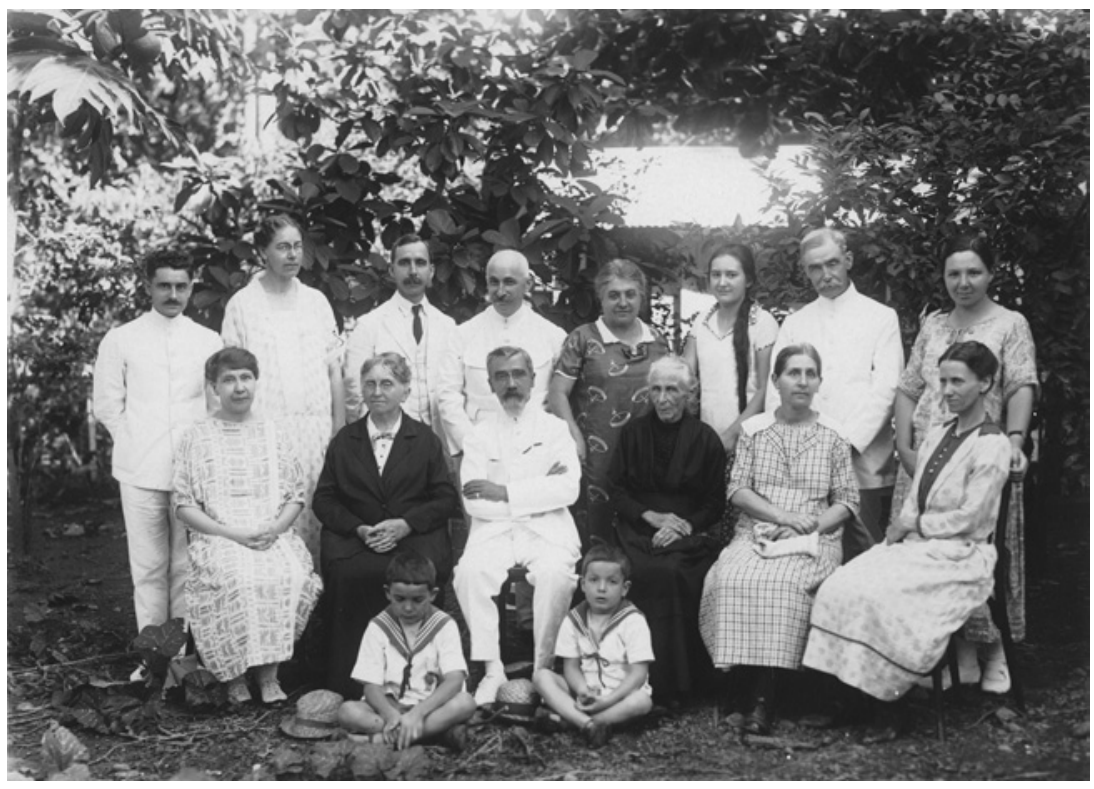

Photo $n^{\circ}$ 1. Personnel missionnaire en 1927, (C) Défap-Service protestant de la mission.

dès 1797 - que politiques : à partir de 1842, date du protectorat français sur Tahiti et Moorea, transformé en annexion en 1880, l'activité des missionnaires britanniques devient difficile. A partir de 1863, les missionnaires de la SMEP doivent donc faire la preuve, vis-à-vis des autorités administratives, qu'il est possible d'être à la fois protestant et patriote français.

Dans l'entre-deux-guerres, Tahiti est décrite comme le plus isolé et le plus éloigné de tous les champs de missions de la SMEP et en même temps comme «le phare » et « la lumière » du Pacifique ${ }^{6}$. En 1928, la population protestante est estimée à 19000 (soit un peu plus de la moitié de la population totale); la Mission de Tahiti ne reçoit pas d'aide financière de la SMEP, elle est financièrement indépendante grâce aux dons

6) «La mission protestante dans les îles du Pacifique», note dactylographiée rédigée en 1928 par Monsieur Ahnne, missionnaire instituteur, bibliothèque du DEFAP, boîte D9 « Le Pacifique ». 
des Polynésiens récoltés à l'occasion du mê (collecte annuelle) et des kermesses des écoles protestantes ${ }^{7}$. Trois missionnaires occidentaux et cinquante à soixante pasteurs indigènes assurent l'encadrement des paroisses polynésiennes à Tahiti-Moorea, aux îles Sous-le-Vent et aux îles Australes. L'évangélisation apparaît intimement liée aux processus de «civilisation » et d'instruction. Si l'occidentalisation des normes et des pratiques est un héritage des missions britanniques, l'implantation d'écoles protestantes à Tahiti et Raiatea, au cours du dernier quart du XIX ${ }^{\mathrm{e}}$ siècle, constitue un élément novateur destiné à entretenir - grâce à l'apprentissage du français qui y est dispensé - de bonnes relations avec les autorités administratives françaises.

Dès l'origine des missions en Océanie au début du XIX ${ }^{\mathrm{e}}$ siècle, les missionnaires protestants ont eu pour objectif de convertir les âmes et de dresser les corps. Richard Eves a montré à partir de l'analyse des missions méthodistes en Papouasie Nouvelle-Guinée l'imbrication entre ces deux objectifs : "Conversion was confirmed in the eyes of the missionaries by the acquisition of a visibly distinct form of body, emblematic of Christian character ${ }^{8}$. En Polynésie, l'historienne Claire Laux a également montré comment la valorisation du modèle de la famille nucléaire s'accompagnait d'une transformation de l'habitat polynésien - les huttes ouvertes sont transformées en maisons en dur fermées - et de l'apprentissage de comportements et de règles morales comme l'intimité et la pudeur ${ }^{9}$. L'inspection des maisons, maintenue aux îles Australes dans l'entredeux-guerres, est l'occasion de rappeler, selon le missionnaire Charles

\footnotetext{
7) L'association scolaire protestante créée officiellement en mai 1921 a pour objet de subvenir aux frais d'entretien, à l'amélioration et à la reconstruction des écoles de la Papeete. (Francis Cheung, Tahiti et ses îles (1919-1945) Etude d'une société coloniale aux antipodes de sa métropole, Paris, l'Harmattan, 1998, p. 397); Les institutrices âgées continuent également à donner des leçons privées payantes et à reverser à la Mission de Tahiti les sommes ainsi récoltées.

8) «As well as being clothed, clean, neat and orderly, this body incorporated appropriate habits, comportments and gestures indicative of a disciplined Christian, whose interior morality was consistent with his or her outer body.» Richard Eves, "Colonialism, Corporeality and Character: Methodist Missions and the Refashioning of Bodies in the Pacific », History and Anthropology, 1996, vol.10 nº 1 , p. 86.

9) Claire Laux, Les théocraties missionnaires en Polynésie (Tahiti, Hawaii, Cook, Tonga, Gambier, Wallis et Futuna) au XIX siècle: des cités de Dieu dans les Mers du Sud? Paris, L'Harmattan, 2000, pp. 145-148.
} 
Vernier, que «cette propreté extérieure devait être le symbole de celle du cœur et de l'âme $»^{10}$.

Dans l'entre-deux-guerres, ce lien ontologique entre apparence extérieure et état moral intérieur est reformulé : le corps à discipliner désigne tout autant des individus qu'une Eglise locale en cours de constitution. Les missionnaires comparent, par analogie, le «jeune âge » de l'Eglise locale instituée par le décret du 5 juillet $1927^{11}$ à la population relativement jeune de Tahiti ${ }^{12}$. Dans une société polynésienne en profonde transformation où la grippe espagnole de 1918-1919 a fait de nombreux morts parmi les cadres de l'Eglise, l'Eglise locale est implicitement décrite sur le mode d'un corps en transformation, celui d'un adolescent qui doit, pour grandir, continuer à bénéficier des enseignements des adultes : en d'autres termes, l'autonomie de l'Eglise locale vis-à-vis de la société missionnaire est sans cesse repoussée à un avenir lointain :

\begin{abstract}
Notre Mission ne revêt plus le même caractère qu'autrefois; l'évolution s'est faite ici comme partout ailleurs. Le pays, en devenant si accessible aux Européens, s'est vu inondé de touristes ou de colons, dont les mœurs faciles ont gâté beaucoup de choses; le dimanche n'est plus respecté comme jadis; notre jeune génération, toujours attachée à l'Evangile, ne l'est cependant pas au même degré que celle qui vient de disparaître, presque complètement, pendant la grippe de la fin de 1918. Elle est encore trop jeune et superficielle...l'œuvre se complique d'année en année, elle devient de plus en plus exigeante; aussi est-ce pour les quelques missionnaires une perpétuelle tension ${ }^{13}$.
\end{abstract}

10) «Tahiti une tournée aux îles Australes» récit de M. le missionnaire Charles Vernier, Journal de la SMEP, juin 1921, pp. 459-461.

11) La loi de du 9 décembre 1905 sur la séparation des Eglises et de l'Etat n'est pas appliquée dans les Etablissement français d'Océanie «pour des raisons d'opportunité locale». Ce décret de 1927 octroie aux Eglises protestantes de Tahiti et des îles une plus grande liberté et une plus grande indépendance. Le ministre des Colonies dans son rapport au président de la République Française du 27 juin 1927 écrit « ces modifications doivent tendre plus particulièrement à supprimer notamment l'intervention de l'Administration dans le fonctionnement interne du culte tout en réservant à celle-ci un droit de regard suffisant ». (Archives nationales d'Outre-mer, Aix en Provence, Fonds ministériels, Océanie).

12) Entre 1920 et 1940 la population de la colonie est passée de 30000 à 50 ooo habitants.

13) Charles Vernier, Journal de la SMEP, mai 1921, Tahiti dernières nouvelles, p. 386. 
A côté de l'évangélisation, la création d'écoles protestantes par les missionnaires français illustre une conception particulière de la place des protestants au sein de la colonie française. L'importance accordée à l'instruction correspond, selon le pasteur et historien Henri Vernier, à la volonté des missionnaires protestants français d'inscrire leur action dans une perspective de soutien à la France : il s'agit ainsi d'apprendre aux enfants le français sans qu'ils changent de religion, et de faire face à la concurrence de l'Église catholique qui a fondé sa stratégie de conquête sur l'enseignement ${ }^{14}$. A la fin du XIXe siècle, la place que doit tenir l'œuvre scolaire dans la Mission protestante divise les missionnaires protestants. Frédéric Vernier prône à la fois un développement des écoles protestantes et une participation active des missionnaires à la vie politique locale. En revanche, certains de ses collègues refusent à la fois la priorité - notamment financière - donnée à l'œuvre scolaire protestante et une quelconque implication de la part des missionnaires dans la vie politique locale, jugeant nécessaire un recentrage de l'action missionnaire sur l'évangélisation. L'historienne Annick Lombardini souligne que la question de la langue de l'enseignement dispensé dans les écoles protestantes divise également les missionnaires: les pasteurs souhaitent privilégier la langue locale (la Bible ayant été traduite en tahitien en 1838), alors que les instituteurs comme François Atger veulent enseigner le français afin d'éveiller le sentiment d'appartenance à la nation française ${ }^{15}$.

La mise en place, à partir de 1864 , de ces écoles protestantes vise en outre à former les futures élites du pays ${ }^{16}$. Elle s'accompagne d'une scolarisation différenciée selon les sexes: les jeunes filles semblent l'objet de toutes les attentions, elles sont plus surveillées, l'école étant présentée comme un moyen de les éloigner des «dangers du monde ${ }^{17}$. Surtout, les jeunes filles sont perçues comme le maillon permettant d'atteindre

\footnotetext{
14) Jean-François Zorn, Le grand siècle d'une mission protestante. La Mission de Paris de 1822 à 1914, Paris, Karthala \& Les Bergers et les Mages, 1993, p. 211.

15) Annick Lombardini, «L'influence socio-politique de l'Eglise protestante dans la société polynésienne de 1863 à 2003», Thèse d'histoire, Université de Polynésie française, 2003, pp. 66-71.

16) Henri Vernier, Au vent des cyclones, missions protestantes et Eglise évangélique à Tahiti et en Polynésie française, Papeete, Eglise évangélique de Polynésie française, 1986, p. 114.

17) JSMEP, 1891, p. 444.
} 
l'ensemble de la cellule familiale. Selon la pensée du pasteur Charles Viénot, «la création d'une forte école de jeunes filles était, entre toutes, l'œuvre à faire pour préserver la population féminine de Taïti et pour exercer sur la nation toute entière une influence chrétienne $»^{18}$. Les effectifs des écoles protestantes permettent de mesurer cette attention particulière ${ }^{19}$. En 1882, lors de la remise des prix, le pasteur Vernier explique comment la mission doit exercer une influence sur les jeunes filles :

\begin{abstract}
Appelées un jour, suivant l'ordre de la nature, à devenir épouses et mères, nous voudrions que vous puissiez porter à votre foyer, avec le trésor intact de vos affections, toutes les vertus qui doivent en faire l'ornement, à savoir, le goût du travail, de l'ordre, de l'économie, le savoir-vivre, la sagesse, la piété, la fidélité, etc., vertus qui seules vous permettront de trouver dans la pratique journalière des devoirs domestiques, le bonheur que tant d'autres de vos amies cherchent hélas! dans les honteuses jouissances de l'oisiveté. (...) votre bonheur, présent et futur, tel est donc le but à atteindre ; le devoir par le travail et la piété, tel est aussi le moyen ${ }^{20}$.
\end{abstract}

La priorité accordée à l'éducation des jeunes filles - observable également au sein de la mission catholique ${ }^{21}$ - nécessitait la venue d'institutrices diplômées envoyées par la SMEP. C'est en 1885 qu'arrive la première d'entre-elles : mademoiselle Emilie Banzet. En Océanie, l'historien Charles Forman a montré que c'est au cours du dernier quart du XIXe siècle que le champ missionnaire permit à des femmes occidentales, de plus en plus nombreuses à partir à l'étranger, d'accéder à des postes à responsabilité, particulièrement dans l'enseignement ${ }^{22}$. Mais cette féminisation des

18) JSMEP, 1905, p. 208.

19) En 1890-1891, les écoles de Papeete comptent 92 garçons scolarisés sur un total de 230 élèves (JSMEP, 1891, p. 207). En 1910, l'école des garçons de Papeete compte 180 inscrits (avec une moyenne de présence journalière de 120 à 130), l'école des filles compte 314 élèves (JSMEP, 1910, 447-448); en 1920 l'école comprend (approximativement) 250 garçons et 300 filles (JSMEP, 1920, pp. 221-222); les chiffres sont respectivement de 250 et 325 en 1930 (JSMEP, 1931, pp. 203-204).

20) JSMEP, 1884, pp. 482-483.

21) Pour une analyse détaillée de l'action de la congrégation des sœurs de Saint Joseph à Tahiti et aux Marquises: James Patrick Daughton, An Empire Divided: Religion, Republicanism, and the Making of French Colonialism, 1880-1914, New York, Oxford University Press, 2006, pp. 121-144.

22) Charles Forman, « Sing to the Lord a New Song » : Women in the Churches of Oceania », in Denise O'Brien et W. Tiffany Sharon (eds.), Rethinking Women's Roles. Perspectives from the Pacific, University of California Press, 1984, pp. 153-172. 
organisations missionnaires, liée à la professionnalisation de leurs activités, ne s'est paradoxalement pas traduite, à Tahiti, par un essor des publications des femmes missionnaires.

\section{Ecritures missionnaires, écritures féminines}

Chantal Paisant, à propos des correspondances des sœurs catholiques en mission à l'étranger, rappelle la diversité des formes d'écriture dépendant à la fois de l'époque, du statut et du niveau d'instruction des auteures et de leurs destinataires : correspondances réservées à la supérieure générale, à la congrégation ou à un lectorat extérieur ${ }^{23}$. Dans ce dernier cas, l'objectif de promouvoir la mission auprès d'un public élargi afin de récolter des fonds incita les sœurs à s'éloigner du registre de l'intime pour privilégier une écriture factuelle et impersonnelle ${ }^{24}$.

En milieu protestant francophone, les écrits - et notamment les écrits publiés - des missionnaires à Tahiti sont dans la grande majorité des cas des écrits d'hommes : il s'agit de mémoires ${ }^{25}$, de lettres et comptes rendus adressés à la direction de la SMEP parfois publiés dans le journal de la SMEP (JSMEP). Ce journal destiné à un lectorat protestant a pour objectif de rendre compte de l'avancée de l'œuvre missionnaire, à l'étranger et dans les colonies, et de son nécessaire soutien. De nombreux appels aux vocations missionnaires sont publiés, destinés à pallier le manque de personnel à Tahiti et dans les îles polynésiennes. Pour être publiables, les lettres (ou extraits de lettres) doivent implicitement répondre à plusieurs critères : donner l'impression d'une œuvre en expansion qui malgré ses succès - ou à cause d'eux - continue à avoir besoin de l'aide financière et humaine de la SMEP. Les lettres doivent également éviter d'aborder des conflits personnels et trouver un juste équilibre entre une description précise des conditions de vie en Polynésie et des réflexions d'ordre plus général. Cet exercice

\footnotetext{
23) Chantal Paisant, La mission au féminin. Anthologie de textes missionnaires, Turnhout (Belgique), Brepols, 2009, pp. 25-28.

24) Ibid.

25) Parmi les mémoires publiés des missionnaires à Tahiti : Thomas Arbousset, Tahiti et les îles adjacentes. Voyages et séjour dans ces îles, de 1862 à 1865, Paris, Grassart, 1867, 323p. André Vernier, Souvenirs d'Océanie, Paris, Société des Missions Evangéliques, 1942, 115p. Charles Vernier, Tahitiens d'hier et d'aujourd'hui, Paris, Société des missions évangéliques, 1948, 287p.
} 
d'écriture est en réalité difficile. D'une part les missionnaires sont surchargés de travail et ne trouvent pas toujours le temps d'écrire. En janvier 1926, le lecteur du Journal de la SMEP peut ainsi lire : « Rares sont les nouvelles de Tahiti dans le Journal des Missions, depuis quelque temps. On pourrait en conclure que nous n'avons rien d'intéressant à raconter. En vérité, les faits ne manquent pas ; c'est bien le temps qui fait défaut ${ }^{26}$. D'autre part, le devoir de rendre compte le plus fidèlement possible de l'œuvre missionnaire s'accommode mal d'une euphémisation des problèmes personnels ou communautaires nécessaire à toute publication. Mademoiselle Spelta, institutrice à l'école mixte de Raiatea (Iles sous-le-Vent) résume les difficultés de cette écriture codifiée dans une lettre du 7 novembre 1929 adressée à Monsieur Barnaud à Paris :

\begin{abstract}
Votre reproche à propos de notre manque de correspondance me touche au vif, car moi-même je me fais souvent des reproches à ce sujet. Croyez, cependant, que pour nous il est bien difficile de trouver des sujets dignes d'intérêt et qui puissent être publiés. Notre champ est petit ; plusieurs sont les personnes qui reçoivent les journaux missionnaires, par conséquent certaines choses ne peuvent pas être imprimées, sous peine de fausse interprétation. Toutefois, je veux essayer d'écrire quelque chose que vous puissiez faire paraître, sans faire de tort à personne.
\end{abstract}

Surchargés de travail ou incapables de répondre aux injonctions contradictoires de cette pratique d'écriture encadrée, les missionnaires, et notamment les femmes, sont peu nombreux à écrire autre chose que les comptes rendus annuels obligatoires. Et les missionnaires en viennent à se demander si l'absence d'intérêt en Métropole pour la mission de Tahiti ne viendrait pas «d'un défaut de contact épistolaire suivi entre les missionnaires et ceux qui les ont envoyés? $\gg^{27}$.

Si les femmes missionnaires sont peu nombreuses à publier dans le Journal de la SMEP, en revanche les correspondances privées que ces femmes missionnaires institutrices ou infirmières adressent aux directeurs de la SMEP à Paris permettent de repérer des pratiques d'écriture féminine. Alors que Monsieur Allégret, directeur de la SMEP, est en mission à Tahiti

26) Remarque introductive de la lettre de M. le missionnaire Ch. Vernier du 10 janvier 1926 (Tahiti) publié dans le Journal de la SMEP, 1926, p. 182.

27) Rapport de la Mission de Tahiti, exercice 1929-1930, JSMEP, mars 1931, p. 200. 
d'août 1926 à juillet 1927, les institutrices continuent en certaines occasions à lui écrire et justifient ainsi leur démarche :

Cher Monsieur, Etre si proche et écrire alors que lorsque vous étiez bien plus loin, je ne l'ai guère fait! Mais d'une part, c'est un moyen plus économe de temps; et vous ici, vous pouvez demander de vive voix toutes explications nécessaires [...] (Mademoiselle Emma Robert, lettre du 31 août 1926).

Je vous envoie ce mot parce que je ne peux pas vous voir avant l'école [...]. Je vous écris aussi pour formuler ma pensée plus nettement que je n'aurais pu le faire de vive voix (Mademoiselle Emilie Banzet, 20 novembre 1926).

Ces femmes écrivent donc ce qu'elles n'ont pas osé (ou pu) dire de vive voix. Ces pratique d'écriture attestent la hiérarchie des rapports de genre et de pouvoir au sein de la mission protestante à Tahiti : les institutrices s'estiment moins légitimes à prendre la parole en public, notamment lors des réunions entre missionnaires organisées à l'occasion de la venue de Monsieur Allégret, même si dans le même temps, lorsque les discussions portent sur les écoles protestantes, ces femmes se doivent de donner leur point de vue. Cette moindre légitimité est attestée à la fois par les statuts de l'Eglise et par les choix de publication opérés par le directeur du journal de la SMEP. Jusqu'en 1927, les femmes missionnaires ne peuvent siéger au sein de la Conférence de Tahiti, instance décisionnelle de l'Eglise. Cette moindre légitimité se donne également à lire dans le Journal de la SMEP où sont privilégiés des extraits de lettres qui attestent du respect des hiérarchies de statut et de genre dans le champ missionnaire ; c'est notamment le cas en octobre 1927 où les lecteurs du Journal de la SMEP peuvent lire un extrait d'une lettre de Mademoiselle Banzet évoquant le retour en Métropole du directeur de la Société :

M. Allégret nous a quittés. Nous nous sentons orphelins. C'était une telle sécurité de le sentir là, toujours prêt à solutionner les questions difficiles, à soutenir ceux qui faiblissaient. D’autres plus autorisés, mieux qualifiés, diront ce qu'il a été pour notre oeuvre à Tahiti ${ }^{28}$.

Mais la lecture des correspondances privées suggère aussi une autre forme d'écriture, plus rare, alliant affirmation de soi et contestation de l'autorité

28) JSMEP, octobre 1927, p. 602. 
hiérarchique. Mlle Lafon, institutrice à l'école mixte de Raiatea, écrit à M. Allégret qu'elle refuse sa nouvelle affectation à Papeete :

\begin{abstract}
J'ai été tellement prise par surprise par ce que vous m'avez dit que je ne vous ai pas répondu comme je désire le faire. Si j'étais plus jeune peut-être courberais-je la tête et accepterais-je ce que vous semblez avoir décidé. A mon âge on ose tout et je vous dirai très franchement ce que je pense. C'est la première fois de ma vie que je vois mon sort décidé sans que j'aie eu connaissance des raisons qui vous poussent à agir ainsi. Il est dit que tout accusé doit pouvoir se défendre. Qu'ai-je fait, ou que n'ai-je pas fait, pour vous donner l'idée que je ne suis pas à ma place à Raiatea? [...] Vous avez l'air de penser, cher Monsieur, que je ne m'occupe pas assez de l'église. J'aimerais beaucoup m'en occuper, mais quand ? [...] Non, évidemment, j'ai fait ce que j'ai pu. Si cela n'est pas assez, comme je vous l'ai déjà dit, cher monsieur, la solution est très simple. Disons qu'à la fin de l'année scolaire, en juillet, je donne ma démission, je retourne en France. [...] Il est inutile de me demander de venir à Papeete. Je n'accepterai pas. J'aimerais si possible que ma lettre fût lue en Conférence, je désire que tous soient au courant de mes pensées ${ }^{29}$.
\end{abstract}

Entre écritures attestataires et contestataires, les correspondances privées permettent également, en comparant ces correspondances aux écrits publiés par le Journal de la SMEP, de mettre au jour les silences volontaires de la direction de la Société. Si Mademoiselle Lafon « n'est pas à [s]a place » à Raiatea, c'est parce qu'elle ne s'occupe pas suffisamment, selon sa collègue également institutrice, des paroisses protestantes de l'île qui en l'absence de missionnaire métropolitain sont dirigées par des pasteurs polynésiens sous le contrôle officieux des femmes missionnaires. Bien que non consacrées pasteurs, certaines femmes missionnaires célibataires, institutrices ou infirmières, ont donc eu des activités pastorales sortant d'une répartition traditionnelle des tâches selon le sexe, qui attribue aux hommes missionnaires l'évangélisation et la prédication, aux femmes missionnaires et épouses de missionnaires, l'éducation des enfants et le soin au malades. C'est à Raiatea, en l'absence de missionnaire métropolitain, que les femmes institutrices de l'école mixte de Uturoa ont dû prendre en charge, au cours des années 1920-1930, une partie des activités réservées aux missionnaires, sans que ces activités n'apparaissent jamais dans le Journal de la SMEP30.

29) Lettre de Marguerite Lafon du 21 octobre 1926 (Uturoa) adressé à Monsieur Allégret.

30) Pour une analyse des activités pastorales des institutrices à Raiatea, voir les lettres de Mademoiselle Spelta, notamment celle du 11 janvier 1928 adressée à Monsieur Couve. 
Anna Johnston, pour expliquer la prédominance des écrits masculins parmi les missionnaires de la London Missionary Society au cours de la première moitié du XIX ${ }^{\mathrm{e}}$ siècle, écrit : « in Polynesia and Australia [...] male missionaries, who where the only individuals formally invested with the authority of the societies in this period, mostly monopolised the textual archive and official publications $»^{31}$. De façon comparable, les silences $d u$ journal de la SMEP rappellent que la position sociale d'infériorité des femmes au sein de la mission de Tahiti eut une incidence directe sur le contenu des publications missionnaires et les pratiques d'écriture.

Quelles sont les spécificités du journal d'Anne-Pauline Gilbert et en quoi sont-elles liées à sa position sociale dans l'organisation missionnaire? Dans quelle mesure et jusqu'à quel point ce journal se conforme-t-il ou déroge-til aux pratiques d'écritures missionnaires et féminines?

Anne-Pauline Gilbert embarque à Marseille sur le paquebot Andromède avec Mademoiselle Emilie Banzet, institutrice de l'école des jeunes filles de Papeete qui a contribué à son recrutement et l'a préparée à ce qui l'attend à Tahiti. Elles partent de Marseille le 26 mars 1926 et arrivent à Tahiti le 6 mai, après 43 jours de voyage en mer, la traversée de l'Atlantique, une escale de deux jours à la Guadeloupe et à la Martinique, le passage du canal de Panama et la traversée du Pacifique jusquà Tahiti en passant par les Tuamotu. On sait peu de choses du passé d'Anne-Pauline Gilbert : elle est originaire de Mulhouse, elle n'a pas de famille ; elle a sans doute été aidesoignante pendant la première guerre mondiale; c'est à la lecture des lettres adressées au directeur de la SMEP qu'on apprend que Mademoiselle Gilbert est née en 1877 : elle a donc 49 ans quand elle part à Tahiti en $1926^{32}$. Mademoiselle Anne-Pauline Gilbert tient un journal intitulé « Récit de voyage et de premières impressions d'une institutrice missionnaire à Tahiti » pendant les dix-huit premiers mois, de mars 1926 à août 1927. Ce journal est interrompu de façon temporaire (de septembre à décembre 1926 et de mai à juillet 1927) puis définitive, en août 1927, sans doute par manque de temps et par lassitude. Ce Journal s'adresse à une trentaine d'amies et prend la forme de lettres dont certaines sont redistribuées. L'objectif apparaît multiple: raconter ses aventures personnelles, faire

\footnotetext{
31) Anna Jonhston, Missionary Writing and Empire, 1800-1860, Cambridge, Cambridge University Press, 2003, pp. 7-8.

32) Lettre du 30 avril 1928 adressée à Monsieur le Directeur.
} 
partager ses impressions et réflexions et éventuellement susciter des vocations missionnaires. Ce Journal, conservé à la bibliothèque du DEFAP 33 sous forme dactylographiée et annotée, s'ouvre sur la note préliminaire suivante :

Ces feuillets ne sont en aucune façon destinés à la publicité. Nous, les institutrices de l'école Viénot, ne désirons pas que nos impressions soient connues, hormis du cercle de nos amis personnels et de celui des amis de l'œuvre qui s'intéressent personnellement à nous. Les appréciations d'une nouvelle venue n'ont d'ailleurs pas de valeur absolue. Que nos lecteurs veuillent donc considérer ce «Journal» comme lettre privée.

Ce «journal » est à mi-chemin entre le journal intime, le récit de voyage et le compte rendu missionnaire. Il prend par moments l'aspect d'un journal intime. Jusqu'alors réservé aux filles des milieux aristocratiques, le journal intime se diffuse, selon Philippe Lejeune, à partir de la seconde moitié du $\mathrm{XIX}^{\mathrm{e}}$ siècle dans les autres classes sociales grâce notamment au développement de l'enseignement secondaire féminin. Tenir un journal intime devait contribuer à l'apprentissage de l'écriture et parfaire l'éducation morale des jeunes filles (notamment au moment de leur première communion) ${ }^{34}$. Agnès Fine souligne la permanence du lien entre écriture de soi et production du féminin à l'époque contemporaine: le journal intime vise aujourd'hui chez les jeunes adolescentes le développement «d'une vie intérieure marquée par la culture des sentiments » et d'une « disposition à ressentir ${ }^{35}$. Anne-Pauline Gilbert note ainsi régulièrement ses impressions, ses sentiments et ses états d'âme. Mais alors que le journal intime est, en règle générale, une écriture d'attente et d'expression des rêveries amoureuses adolescentes qui prend fin avec le mariage, Anne-Pauline Gilbert, célibataire âgée de 49 ans, démarre son journal avec le récit du voyage en bateau vers Tahiti sur le mode de l'aventure et de la découverte.

\footnotetext{
33) Le Defap-Service protestant de mission (Paris) est l'héritier de la SMEP (1822-1971).

34) Philippe Lejeune, Le Moi des demoiselles : enquête sur le journal de jeune fille, Paris, éditions du Seuil, 1993, $454 \mathrm{p}$.

35) Agnès Fine, «La production du féminin. L'exemple de l'écriture de soi » in Irène Théry, Pascale Bonnemère, Laura Lee Downs et Anne-Christine Taylor (eds), Ce que le genre fait aux personnes, Paris, Editions de l'Ehess, 2008, pp. 235-254.
} 
La seconde influence perceptible dans ce journal est en effet celle des romans de voyage ou des «voyages témoignages» qui se développent, à partir de la seconde moitié du XIXe siècle, grâce au développement des transports maritimes permettant d'effectuer des voyages individuels en dehors de missions officielles : ce type de récit qui lie l'aventure à la pédagogie «tient du journalisme de reportage le goût du détail, de la formule souvent percutante, la chasse au pittoresque, comme si les auteurs étaient de vrais explorateurs» note Daniel Margueron ${ }^{36}$. Le journal d'AnnePauline Gilbert témoigne de la volonté de l'auteure d'inscrire ses pas dans ceux des premiers explorateurs et des premiers missionnaires de la London Missionary Society : comme eux, elle se fixe comme objectif d'apprendre la langue tahitienne sur le bateau, et elle découvre, comme eux, que «cette langue tahitienne se révèle bien plus compliquée que je ne le pensais» (p. 14). Anne-Pauline Gilbert relate également ses excursions, notamment lors des escales aux Antilles, sur un mode enjoué, parfois comique ou pittoresque, en imitant les bruits, comme dans les livres d'aventures pour enfants ; elle évoque d'ailleurs Jules Verne au cours de la traversée du canal de Panama: «ici, le paysage évoque soudain aux yeux de ma mémoire les belles reliures de «Jules Verne» de mon enfance, avec leurs couvertures frappées d'or et d'argent» (p. 41).

Enfin ce journal emprunte aux formes codifiées des écritures missionnaires publiées dans le Journal de la SMEP. Il n'est pas fait ouvertement mention de dissensions au sein de la mission protestante : ce journal est de ce point de vue plus proche des lettres «publiables» que des correspondances privées adressées au directeur de la SMEP. Surtout, il affiche un objectif similaire au journal de la SMEP, celui de susciter les vocations comme le rappelle l'auteure en conclusion de son journal: «Si j'ai pu encourager quelque jeune personne à choisir la carrière missionnaire, j'en serai heureuse » (p. 156).

Ce journal apparaît en définitive comme un objet littéraire hybride - à la croisée du fictif et du véridique, à la jonction entre l'intime et le public - lié au statut lui-même ambigu d'Anne-Pauline Gilbert, femme missionnaire bénévole de la SMEP.

\footnotetext{
36) Daniel Margueron, Tahiti dans toute sa littérature, Paris, L'Harmattan, 1989, pp. 64-65.
} 
Le terme de missionnaire désigne à un premier niveau les hommes métropolitains (plus rarement suisses) consacrés ${ }^{37}$. Il désigne, à un second niveau, tous ceux qui sont «envoyés» par la SMEP sans être consacrés. Anne-Pauline Gilbert peut donc être considérée comme une missionnaire dans ce second sens : elle est d'ailleurs désignée orometua api ou « missionnaire nouvelle » lors de sa présentation officielle à la paroisse polynésienne de Paofai à Papeete en mai 1926 (p. 63). Elle se qualifie également dans son journal d'institutrice ou d'éducatrice. Mais c'est à la lecture de la correspondance établie avec les différents directeurs de la SMEP qu'on apprend qu'elle n'est pas une institutrice diplômée mais une «intérimaire bénévole». Son style littéraire et les références qu'elle utilise montrent néanmoins qu'elle a reçu une solide éducation. Elle joue habilement de cette ambiguïté : en tant qu'envoyée par la mission de Paris, elle demande une lettre officielle lui permettant de bénéficier, aux Etats-Unis, de réductions dans les transports réservées aux missionnaires ${ }^{38}$; mais puisqu'elle n'est pas officiellement missionnaire, elle n'effectue pas de compte rendu de ses activités :

J'estime qu'une non-missionnaire n'a pas à faire de rapport sur une œuvre, ni, à donner son avis sur elle, j'ai donc toujours observé une grande retenue qui ressemble à de la paresse épistolaire, je le sais. Qu'on me le pardonne! La chanson reste d'ailleurs la même : trop peu de personnel blanc, et missionnaire vraiment, pour une œuvre tellement européanisée comme nos écoles forcées de lutter contre des institutions rivales, bien montées, dans la ville même. Jusqu'à présent on a tenu. Et il y a des «succès moraux » qui relèvent les courages, les volontés. Nous ne voulons donc pas chanter la chanson tout à fait sur le mode mineur !39

\section{L'œuvre scolaire et les paradoxes de l'apprentissage des normes de genre}

Anne-Pauline Gilbert vit essentiellement en compagnie des autres femmes missionnaires de l'école des filles et des filles de l'internat. Il semble que

37) Ce terme n'est pas utilisé pour les pasteurs polynésiens désignés sous le vocable de «pasteurs indigènes » même si à partir de 1928 ces derniers intègrent la Conférence de Tahiti.

38) Lettre du 23 mars 1933 (Mulhouse) adressée à M. le Directeur.

39) Lettre du 19 janvier 1935 (Papeete) adressée M. le Directeur. 
pour elle l'idéal de la mission protestante ne soit pas l'évangélisation mais les soins aux malades apportés par les deux infirmières protestantes travaillant à la léproserie d'Orofara. Les relations avec l'Eglise catholique sont inexistantes; avec les représentants de la colonie, elles sont rares (elle mentionne l'inspecteur chargé d'introduire des cours de gymnastique et la participation des écoles au défilé du 14 juillet). A l'exception des premiers mois où elle effectue quelques excursions en dehors de Papeete et de la visite régulière des infirmières protestantes à la léproserie, Anne-Pauline Gilbert souligne par la suite le relatif isolement des femmes institutrices à Papeete et le contrôle permanent des faits et gestes auquel elles doivent se soumettre: «les dames missionnaires ne sortent pas dans cette société toute faite de gaîté et de rire» (p. 129). Cette situation a une incidence directe sur le contenu de son journal :

Confinée dans l'Ecole, trop nouvelle, trop lourde et maladroite encore pour embrasser de ma compréhension notre œuvre dans son ensemble. Je ne puis vous donner qu'un aperçu d'une de ces cellules : les fillettes dans l'Internat. Le travail des églises, des districts, de propagande ne m'est pas connu. Je ne puis vous parler conversions, formation de pasteurs indigènes, plus tard, peut-être (pp. 115-116).

Les femmes missionnaires institutrices à l'école des jeunes filles de Papeete doivent dispenser un enseignement de qualité et apprendre à ces jeunes filles à devenir de bonnes épouses et mères de famille. La finalité ultime assignée à l'école protestante, former des futures institutrices ou des futures épouses, est l'objet d'âpres discussions et d'interprétations divergentes parmi les missionnaires.

En 1927 l'école des filles de Papeete emploie quatre institutrices missionnaires (Mlle Perrier, directrice, Mlle Debrie, directrice de l'internat, Mlles Gilbert et Béguin) et quatre « aides indigènes ». L'école des filles enregistre 266 inscriptions (la moyenne des présences est de 214); l'école maternelle 146 inscriptions (avec une moyenne des présences de 54 à 65 ); l'internat entre 42 et 45 enfants. Aux examens de fin d'année (certificat d'études et brevet locaux), sur 24 élèves présentées 19 sont reçues ${ }^{40}$.

Il est demandé aux institutrices missionnaires une grande polyvalence puisqu'Anne-Pauline Gilbert note: «Excepté les leçons de sciences, de

40) JSMEP, 1927, p. 121. 
géographie, je suis préposée à tout, calligraphie et piano compris » (p. 74). Deux mois après son arrivée en juillet 1927 elle écrit :

Vacances ! j'en établis le programme: Apprendre le tahitien, m'initier à la dactylographie; réapprendre à...écrire, surtout faire de la ronde, de la bâtarde, me mettre au piano, et sérieusement; revoir toute mon histoire de France; revoir, refaire tous les points de crochet ; écrire à tout le monde ; établir toutes les collections de timbres promises ; finir enfin l'installation de ma chambre ; soigner fourmis et cancrelats : raccommoder tout; m'initier mieux au ménage; voir Papeete et les environs; préparer les leçons du semestre suivant; et plusieurs etc. ...Vacances? (p. 78).

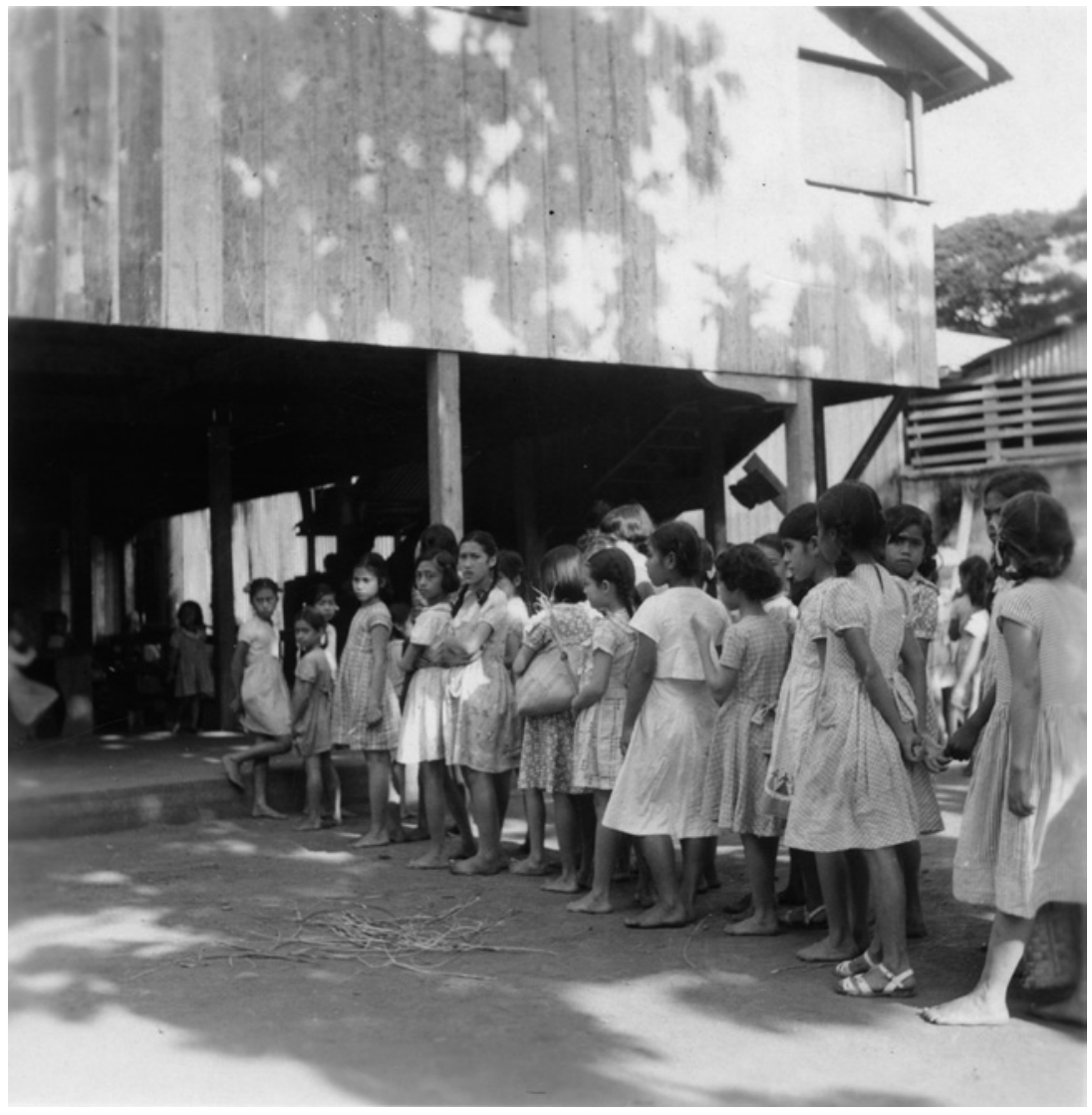

Photo $n^{\circ}$ 2. Ecole des jeunes filles. Papeete (C) Défap-Service protestant de la mission. 
Anne-Pauline Gilbert est aussi chargée de la surveillance de l'internat et, à partir d'août 1927, de la direction de la salle d'Asile, c'est-à-dire de la maternelle, qui scolarise en moyenne entre 40 et 60 enfants, filles et garçons. Les institutrices, qui ont la charge des filles de 6 à 13 ans obligatoirement scolarisées, doivent suivre les programmes officiels et les modifications des programmes leur demandent une préparation accrue ${ }^{41}$.

L'éducation dispensée aux jeunes filles de l'école protestante de Papeete valorise en principe une répartition traditionnelle des tâches selon le sexe. Cette éducation comprend ainsi l'apprentissage de l'entretien du linge pour les plus âgées (p. 66), de la couture et des leçons de piano: «Elles chantent avec facilité, elles dessinent fort joliment et ont de très belles écritures. On les met très tôt à la confection de leur linge, de leurs toilettes et elles s'en tirent bien » (p. 68). Les sorties à l'extérieur de l'école (notamment pour aller au culte) sont encadrées, de même la discipline comportementale imposée aux filles les plus âgées incite la direction de l'école à les dispenser de gymnastique :

Le Commandant de la Place, chargé de - laissez moi dire « améliorer la race » - par le Gouvernement métropolitain, a introduit la gymnastique comme branche d'étude spéciale. [...] A Tahiti, où tout peut dégénérer en frénésie, il faut soigneusement établir un programme de mouvements qui ne doivent rappeler en rien certaines danses douteuses. Nos «grandes», d'emblée sont exclues de ces leçons, et les parents des «moyennes» furent consultées [sic] individuellement au sujet de leurs désirs en la matière (pp. 146-147).

La sexualité des jeunes filles de l'internat est un sujet de préoccupation qu'Anne-Pauline Gilbert évoque brièvement à travers les tûpâpa'u (fantômes, esprits) qui, aux dires des jeunes filles « lancent des pierres » à leurs fenêtres le soir venu (pp. 150-151). Certaines missionnaires institutrices dorment dans le dortoir ou sur la véranda pour dissuader les éventuels prétendants ou voleurs, les tûpâpa'u désignant aussi bien une menace pour la tranquillité de l'esprit, pour les biens matériels de l'Eglise que pour la virginité des filles.

...pour être prête à toute éventualité, j'ai passé deux nuits en plein air sur la véranda où débouche l'escalier central. Là, on est à la portée de tous les appels et sur le passage de

41) Lettre de Mademoiselle Perrier du 30 avril 1928 adressée au directeur de la SMEP. 
«l'homme» le revenant qui, au dire des jeunes filles, se promène sur nos galeries durant les belles nuits tropicales. Inutile de dire que, pas plus que le chef vivant avec ses beaux discours, le chef mort ne me dérange dans mes rêves. Cependant il y aurait de quoi être anxieuse : tous les membres de la Mission qui partent en vacances confient leurs trésors à l'école des filles et ma chambre héberge des biens d'églises, des fortunes privées, des caisses de toute dénomination (p. 110).

Mais l'apprentissage des normes de genre se heurte à un double paradoxe. Le premier est lié à la situation matrimoniale de ces femmes missionnaires qui doivent tout en étant célibataires apprendre à ces jeunes filles à devenir de bonnes épouses et mères de famille. Les femmes missionnaires célibataires doivent vanter les mérites de la maternité, pour ce faire elles redéfinissent les critères de la «bonne mère » en insistant sur le rôle primordial de l'éducation, ce qui leur permet de s'inclure dans cette définition élargie: une bonne mère n'est pas celle qui a des enfants mais celle qui prend soin d'eux et leur donne une bonne éducation ${ }^{42}$. Cette « débiologisation » de la maternité qui peut être comprise comme une transformation radicale de l'injonction à la maternité entre en affinité avec les pratiques polynésiennes extrêmement répandues de l'adoption. L'adoption a suscité des avis divergents de la part des missionnaires, la circulation des enfants étant vue comme un signe de déresponsabilisation des parents biologiques et plus largement comme la preuve du dysfonctionnement des familles polynésiennes; mais certains missionnaires vantent la pratique de l'adoption lorsqu'elle est perçue comme un renforcement de la stabilité et de la solidarité familiale. C'est notamment le cas en 1918-1919: l'épidémie de grippe espagnole a fait 2500 morts, les enfants

42) De façon analogue, Joan F. Burke a montré comment les sœurs catholiques au Zaïre ont redéfini, dans un contexte culturel de valorisation de la maternité et de la fertilité, leur vocation religieuse en termes de maternite spirituelle: «This is in the sense of a motherhood not limited to one family and to the propagation of one clan, but to the bearing and nurturing of life for all the People of God» (p. 264). Joan F. burke, «These Catholic Sisters are all Mamas? Celibacy and the Metaphor of Maternity» in Fiona Bowie, Deborah Kirkwood \& Shirley Ardener (eds), Women and Missions: Past and Present. Antrhopological and Historical Perceptions, Providence/Oxford, Berg, 1993, pp. 251-266. Lire également sur ce sujet: Nancy C. Lutkehaus « Missionary Maternalism: Gendered Images of the Holy Spirit Sisters in Colonial New Guinea » in Mary Taylor Huber \& Nancy Lutkehaus (eds), Gendered Missions, women and men in missionary discourse and practice, University of Michigan Press, 1999, pp. 207-235. 
orphelins ont été adoptés dans le cadre des «familles élargies ». AnnePauline Gilbert estime pour sa part que le système polynésien des adoptions est une bonne chose :

De nombreux enfants restèrent orphelins, parfois seuls au monde, et furent recueillis de droite et de gauche. Nous en avons quelques-uns à l'école de ces enfants de la «grippe » comme on les appelle. L'orphelin tahitien n'est d'ailleurs pas à plaindre. Au contraire de ce que nous faisons en Europe, le Tahitien aime les enfants des autres, non seulement les siens. Il est prêt à toutes les adoptions et le petit adopté a parfois la première place dans l'affection de ses nouveaux parents. Il en prend le nom, ce qui peut mener à une véritable confusion. Au moment des examens, certaines de nos candidates ne s'appelaient pas du tout du nom dont tout le monde les désignait avant l'établissement des actes requis par l'administration (pp. 91-92 $)^{43}$.

Le second paradoxe de l'apprentissage des normes de genre est lié à l'organisation de la vie communautaire à l'internat. L'internat n'apparaît pas comme un lieu idéal d'apprentissage de la domesticité et des normes morales et comportementales telles que la pudeur et l'intimité : les femmes missionnaires et les jeunes filles doivent en effet assurer une multitude de tâches qui les obligent à dépasser une répartition traditionnelle des activités selon le sexe ${ }^{44}$. L'apprentissage de la pudeur et de l'intimité n'apparaît pas comme un objectif prioritaire pour Anne-Pauline Gilbert :

Une correspondante me demande si nous enseignons la pudeur à nos enfants. Non, pas spécialement. Elles ont, malgré leurs jambes toujours nues, beaucoup de décence

\footnotetext{
43) Elle souligne également toutes les difficultés que rencontrent les missionnaires métropolitains à établir durablement une famille en Polynésie, les enfants devenus adolescents devant partir en Métropole poursuivre leurs études (p. 144). A Hawaii, Patricia Grimshaw a bien mis en évidence, à partir de son étude des parcours des premières femmes de missionnaires protestants américains, entre 1826 et 1850, les contradictions du rôle des épouses de missionnaire censées montrer l'exemple dans l'apprentissage de la domesticité mais dont l'activité missionnaire auprès des Polynésiennes s'effectuait au détriment de leur propre vie de famille. (Patricia Grimshaw, Paths of Duty, American Missionary Wives in Nineteenth Century Hawaii, Honolulu, University of Hawaii Press, 1989).

44) Le journal de la SMEP mentionne également quelques (rares) cas de contournement des normes de genre pendant l'entre-deux-guerres. Les enfants scolarisés à l'école des garçons de Papeete doivent ainsi faire la vaisselle : « ...un grand garçon ne voulait pas travailler à la vaisselle sous prétexte que c'était le travail des filles. Voyant les autres le faire joyeusement, il a fini par s'y mettre, lui aussi » (1925, p. 50).
} 
naturelle que nous respectons infiniment. Ainsi, je ne traverse le dortoir que les yeux baissés, et je sens que mes filles m'en savent gré (p. $\left.15^{2}\right)^{45}$.

Ce détournement du regard est aussi celui d'une femme qui remet en cause ne serait-ce que partiellement le rigorisme des missionnaires protestants. Autorisée à assister aux festivités du 14 juillet, elle ne trouve pas les danses particulièrement indécentes : «[...] je dirai tout de suite que je n'ai été ni saisie d'admiration ni scandalisée par le spectacle» (p. 81), alors que ces danses sont interdites par les missionnaires aux membres d'Eglise.

L'avenir des jeunes filles de l'école protestante est l'objet de discussions : peuvent-elles devenir institutrices ou doivent-elles devenir exclusivement de «bonnes épouses » (lâge légal du mariage est fixé à 15 ans pour les filles)? Les élèves ne doutent pas de pouvoir accéder, grâce à l'éducation reçue, à une position professionnelle enviable : « Pour avoir une place aux bureaux ou être un grand homme il faut passer par la grammaire » écrit une jeune fille dans une rédaction de français corrigée par Anne-Pauline Gilbert (p. 114). Les meilleures élèves deviennent assistantes à l'école mais Anne-Pauline Gilbert regrette régulièrement - à cause de la surcharge de travail occasionnée - le départ de ces assistantes «indigènes » de l'école qui partent pour se marier.

La formation scolaire diplômante est une garantie de trouver un emploi rémunéré dans l'administration ou dans les écoles laïques ou protestantes, mais cet emploi est-il compatible avec le mariage alors que le modèle en vigueur dans les écoles protestantes (et dans les écoles laïques jusqu’à la fin du XIXe siècle) est celui d'institutrices célibataires? Le cumul d'une activité professionnelle et d'une vie maritale semble difficile dans la mesure où il pourrait être interprété comme une remise en cause de la hiérarchie des sexes au sein du couple, de ce fait il est l'objet d'avis divergents parmi les missionnaires ${ }^{46}$. Un échange de courrier en 1926 entre mademoiselle

45) Tout en enseignant aux jeunes filles ce détournement du regard, Anne-Pauline Gilbert ne semble pas, sur ce point, écoutée puisqu'elle écrit : «En route, c'est ma robe de soie noire qui défraye la plus grande partie de la conversation. Et lorsque je dis à mes petites : « Mais regardez donc la mer elle est autrement belle que ma robe», elles répondent « oh non? on ne voit pas à travers» (p. 116).

46) Il faut attendre 1946 pour que soit officiellement mentionné dans le JSMEP le cas de femmes polynésiennes mariées et institutrices (JSMEP, janv.-fév.1947, p. 35). 
Emma Robert, institutrice de l'école des jeunes filles, et monsieur Allégret donne un aperçu de ces discussions :

\begin{abstract}
L'autre jour - écrit Emma Robert - lorsque j'ai suggéré comme emploi pour des jeunes filles la carrière d'institutrices, il est évident que je pensais à des institutrices mariées, mais ayant passé dans nos écoles et y ayant reçu une préparation pédagogique. De même que vous entrevoyez des cours spéciaux: sténographie, dactylographie, etc. Il faudrait offrir une préparation spéciale aux futures institutrices (techniquement, religieusement). Il est inutile de vous écrire sur l'influence immense de ceux qui instruisent sur le peuple ${ }^{47}$.
\end{abstract}

En d'autres termes, pour légitimer sa prise de position en faveur de l'accès des femmes polynésiennes au métier d'institutrice, Emma Robert est obligée d'inscrire cette revendication dans le respect des normes maritales (l'obligation de mariage) et des normes de genre (spécialisation en sténographie et en dactylographie) traditionnelles. Elle envisageait sans doute que ces jeunes filles puissent devenir institutrices célibataires sur le modèle des femmes missionnaires, au parcours à la fois exceptionnel (comme l'atteste leur célibat) et exemplaire (aux yeux des jeunes filles scolarisées). Or le directeur de la SMEP ne semble envisager le célibat et la professionnalisation que pour les femmes occidentales.

Au delà des logiques missionnaires, ce débat atteste aussi la transformation de l'activité des femmes en France métropolitaine, dans le cadre du développement du secteur tertiaire, associée au maintien de la différence entre les sexes. Comme le rappelle l'historienne Françoise Battiagliola: « $\mathrm{Au}$ début $\mathrm{du}\left[\mathrm{xx}^{\mathrm{e}}\right]$ siècle, l'exercice d'une profession était généralement lié au célibat: les postières, employées ou enseignantes restaient célibataires [...] Selon Anne-Marie Sohn, cette relation entre exercice d'une profession et célibat se distend entre les deux guerres ${ }^{48}$. A la fin du XIX siècle, l'administration, inquiète du célibat des institutrices, encourage les mariages entre enseignants : «D’apostolat laïque impliquant le célibat, le métier d'institutrice apparaît ainsi dès l'entre-deux-guerres comme particulièrement compatible avec les rôles d'épouse et de mère dans la sphère privée ${ }^{49}$.

\footnotetext{
47) Lettre du 31 août 1926.

48) Françoise Battagliola, Histoire du travail des femmes, Paris, La découverte, 2008, p. 60.

49) Ibid, p. 67.
} 
La lecture du Journal de la SMEP - et de celui d'Anne-Pauline Gilbert suggère que si des jeunes filles diplômées ont pu exercer en tant qu'institutrices adjointes dans les écoles protestantes, elles ont dû interrompre leur activité au moment du mariage. Dans le même temps, les instituteurs du cadre local des écoles protestantes sont, à de rares exceptions près, des institutrices. La faible rémunération est systématiquement mentionnée pour expliquer le peu d'attrait que la fonction exerce auprès des hommes polynésiens qui, lorsqu'ils sont diplômés, choisissent des professions mieux rémunérées ${ }^{50}$. A l'inverse, cette faible rémunération n'est pas incompatible avec l'emploi féminin, à mi-chemin entre l'activité bénévole et le travail salarié.

L'instruction constitue donc moins pour les jeunes filles scolarisées une émancipation par le travail salarié qu'une « dot » destinée à faire un «beau mariage ». C'est ce que suggère Anne-Pauline Gilbert lorsqu'elle évoque les mariages des « aides indigènes » de l'école :

Ce qu'il y a de joli dans ces mariages, c'est que l'amour seul les inspire. Nos filles n'ont point d'argent, pas de famille influente. Elles n'auront jamais besoin de se dire : «mon mari n'aurait pas voulu ou pas pu (ce qui revient au même), m'épouser, si je n'avais ma dot et mes attaches». Ah! comme on les embrasse de bon cœur, nos filles aux yeux rayonnants! Les trois emporteront un solide fonds religieux (p. 150 $)^{51}$.

Dans le même temps, les critères définissant un «beau mariage » apparaissent en définitive limités : «Par mari il faut entendre un époux sérieux, protestant de préférence, donnant des garanties d'existence honnête» (p. 92).

Ces discussions concernant l'objectif assigné à l'éducation des jeunes filles, entre travail salarié et valorisation du modèle de la «bonne épouse », doivent être resituées dans le cadre plus large de la politique missionnaire en matière sexuelle et des débats relatifs à la reconnaissance des divorces. Alors qu'Anne-Pauline Gilbert vante les mérites du mariage d'amour, la

50) Rapport annuel de la Mission de Tahiti, année 1926, publié dans le Journal de la SMEP, 1927, p. 325 .

51) On peut néanmoins se demander si cette vision romantique et sentimentale du mariage d'Anne-Pauline Gilbert est partagée par ces jeunes filles lorsqu'il s'agit de «mariages arrangés » par les parents. 
littérature missionnaire décrit différemment les spécificités du mariage en Polynésie.

En Polynésie, où la liberté sexuelle des femmes a été vantée par les premiers circumnavigateurs dès la fin du XVIII ${ }^{\mathrm{e}}$ siècle, les missionnaires protestants de la LMS et de la SMEP ont fait de l'obligation de mariage - et de la condamnation des relations sexuelles hors mariage - un élément central de leur politique d'évangélisation. En obligeant les futurs membres d'Eglise (c'est-à-dire ceux qui se préparent à la confirmation du baptême) à se marier, la discipline de l'Eglise lie la profondeur de la foi chrétienne à la sincérité et la fidélité conjugales. Il s'agit par un comportement conjugal exemplaire d'attester de sa foi protestante ${ }^{52}$. Les missionnaires français regrettent pourtant régulièrement le faible nombre de mariages parmi les Polynésiens, laissant supposer que la politique missionnaire est, dans ce domaine, un échec. Ils expliquent ces comportements par le fait que «l'échelle des valeurs morales ne peut être la même pour eux que pour nous ${ }^{53}$ mais aussi par la peur de l'engagement, c'est-à-dire en définitive par un profond respect du caractère sacré du mariage lié, à leurs yeux, au schéma d'une conversion progressive, par étapes :

La Conversion existe-t-elle chez le Tahitien ? qui pourrait le dire? en tout cas elle ne semble pas avoir de rapport avec cette lutte parfois tragique de l'âme qui se rend.[...] Le mot de conversion ne peut s'appliquer qu'à cet effort que font certains vers un mieux, vers une vie plus pure et plus sainte, à l'instigation, d'un pasteur, d'un diacre ou d'un chrétien plus avancé dans la vie spirituelle. Le point de départ de cette évolution semble être le mariage qui n'est très souvent qu'une régularisation. Le couple prend le sentiment de sa dignité, il y a des enfants, l'exemple à donner, la vie à gagner, une nouvelle orientation. Souvent il y a des rechutes mais il en reste toutefois un certain remords qui facilite la repentance. Ce changement de vie si lent qu'il soit, ne peut-il s'appeler la conversion ${ }^{54}$

Comment concilier ces deux conceptions du mariage: le «mariage d'amour» pour les jeunes filles de l'école protestante décrit par AnnePauline Gilbert et «le mariage de régularisation » après plusieurs années

52) Gwendoline Malogne-Fer, Les femmes dans l'église protestante mâohi. Religion, genre et pouvoir en Polynésie française, Paris, Karthala, 2007, pp. 31-35.

53) Journal de la SMEP, mars 1930, propos de Monsieur Moreau, p. 177.

54) Rapport annuel de la Conférence de Tahiti, 1932-1933, JSMEP, décembre 1933, p. 712. 
de concubinage pour le reste de la population polynésienne, si ce n'est en termes d'inégal accès à la sexualité selon le sexe? Le poète Henri Hiro résume dans son mémoire de théologie cette apparente contradiction: « on brandit la virginité comme la plus haute vertu qui puisse émaner du sexe féminin [...] Mais pendant qu'elle s'évertuait à préserver dans la privation, la pureté de son sexe, en rêvant au beau prince, celui-ci se préoccupait à raffiner son art et son goût à conquérir les jupons ${ }^{55}$.

Si le mariage a pour objectif de moraliser les familles polynésiennes, il a aussi une incidence directe sur le fonctionnement ecclésial. En liant mariage et confirmation du baptême, l'Eglise entend contrôler et réintégrer les adultes qui, par le mariage, doivent désormais devenir membres d'Eglise et participer pleinement à la vie paroissiale. Or en 1928 la question de la validation du mariage des personnes divorcées est officiellement posée par la conférence de Tahiti au comité directeur de la SMEP :

\footnotetext{
Question quelque peu étrange, qui, hélas, ne manque pas d'actualité ici....et peut-être aussi dans d'autres Colonies. La question s'est posée dans de tels termes que nous sommes obligés moralement d'avoir recours à vous pour nous départager : Un membre du Conseil Supérieur a adjuré le Conseil d'édicter la règle absolue qu'il soit interdit de bénir le mariage de personnes divorcées, dont le premier conjoint est encore vivant. Il s'appuyait sur Marc X: 11 et 12, passage formel, il faut bien le reconnaître ${ }^{56}$.
}

Mais refuser la bénédiction du mariage de personnes divorcées signifierait que «Dans ce cas, ils ne pourront plus jamais entrer dans l'Eglise. De nous mêmes, nous les maintenons dehors » ${ }^{57}$. Or dans le cas des divorces pour cause d'adultère, celui ou celle qui n'a pas fauté (le plus souvent l'épouse) divorce également et n'est plus membre d'Eglise (il ou elle peut aller au culte mais ne peut prendre ni la sainte cène ni de responsabilités ecclésiales). L'Eglise crée donc les conditions d'exclusion de ses membres y compris parmi ceux qui ne sont pas fautifs. Conscients que cette politique disciplinaire est injuste, notamment à l'égard des épouses, c'est au nom de

55) Henri Hiro, Le mariage en Polynésie, licence de mémoire en théologie, faculté libre de théologie protestante de Montpellier, 1972, p. 97.

56) Lettre dactylographiée de la Conférence de Tahiti du 7 janvier 1928 adressée à Messieurs et Honorés Directeurs, p. 7 .

57) Ibid., p. 8. 
l'égalité des sexes que les missionnaires demandent l'autorisation de reconnaître comme membres d'Eglise les personnes divorcées: «le divorce tel qu'il est prononcé actuellement, n'offre plus le caractère d'abominable partialité qu'il revêtait du temps de JESUS, où la femme était à la merci de l'homme et de ses caprices et de ses colères. Il ne doit plus être prononcé qu'à bon escient $»^{58}$.

Mais une autre raison, non mentionnée dans les comptes rendus publiés dans le Journal de la SMEP, explique l'actualité de ces débats sur le divorce : deux missionnaires occidentaux envoyés par la SMEP sont accusés d'adultère en 1926-1927; ils ne sont pas démis de leur fonction mais changent d'affectation alors que les pasteurs indigènes sont systématiquement démis de leurs fonctions pour les mêmes faits ${ }^{59}$. Comment expliquer cet écart de traitement entre missionnaires européens et pasteurs polynésiens alors que la reconnaissance des divorces est demandée au nom de la promotion de l'égalité des sexes parmi les membres d'Eglise polynésiens? Pour les missionnaires européens l'adultère est un acte répréhensible remettant - en principe - en cause l'exercice d'un ministère d'autorité au sein de l'Eglise sans remettre en cause la sincérité de la foi protestante; en revanche pour les Polynésiens l'adultère est une remise en cause de la

\footnotetext{
58) Ibid., p. 8.

59) Monsieur René Pailloux, arrivé à Tahiti en 1924 en tant que missionnaire instituteur, devient, après son divorce, salarié (à hauteur de goo francs) à l'école des garçons de Papeete « au titre purement local» jusqu'en 1930, date à laquelle les relations avec la Mission de Tahiti se dégradent (Lettre de la Conférence de Tahiti du 28 août 1930). Monsieur René Lövy rentre en Métropole et devient pasteur de la paroisse de Bordeaux. C'est lors de son éventuel retour à Tahiti que Charles Vernier, au nom de la Conférence de Tahiti, écrit aux directeurs de la SMEP : "Comment pourrait-il lui-même administrer des communautés chrétiennes qui pourraient un jour lui en faire un reproche public ? Et alors, quelle situation intenable que la sienne et la nôtre? Et alors aussi, quel renom serait celui de la Société des missions de Paris, bien ternie déjà dans certains milieux, depuis le divorce Pailloux. Non? nous ne pouvons pas risquer une nouvelle expérience, qui pourrait être désastreuse, nous le voyons bien. Et puis, que diraient quelques-uns de nos anciens pasteurs indigènes que nous avons dû destituer complètement à cause de la même faute. Il ne comprendraient pas et l'on nous poserait certainement la question directe, avant longtemps. Il ne peut y avoir deux lois dans nos églises tahitiennes: l'une pour le missionnaire et l'autre pour les pasteurs indigènes. C'est extrêmement délicat, vous le sentez vous-mêmes » (lettre du 17 août 1936).
} 
sincérité de la foi protestante qui, aux yeux des missionnaires, se donne à voir en actes.

La promotion de l'égalité des sexes - fondée sur le constat d'une inégalité qu'il convient de corriger - est donc mobilisée pour reconnaître religieusement le divorce des Polynésiens, mais dans les faits le divorce n'entraîne pas les mêmes sanctions pour les missionnaires occidentaux et les pasteurs polynésiens, seuls ces derniers étant démis de leurs fonctions. L'inégalité des sexes permet donc de penser les inégalités «de race » ou pour reprendre l'analyse d'Ann-Laura Stoler «le sexe n'était pas un mécanisme niveleur, mais un espace d'expression et de manifestation des asymétries sociales ${ }^{60}$. Le traitement «privilégié » accordé aux missionnaires occidentaux ne peut donc se comprendre qu'en tenant compte du caractère racialisé et sexué de la mission protestante, elle même liée, parfois malgré elle, à la « mission civilisatrice » de la France en Polynésie.

\section{De la « mission civilisatrice » à la mission réparatrice}

L'intensification de la politique coloniale de la France au cours du dernier quart du XIX ${ }^{\mathrm{e}}$ siècle est légitimée au nom de «la mission civilisatrice » qui est présentée comme un héritage de la Révolution française. La France, patrie des droits de l'Homme, a pour mission d'exporter les principes de 1789 comme le rappelle le gouverneur dans son allocation du 14 juillet 1921 :

Au cours de l'allocution que j'ai prononcée à cette occasion, j'ai fait ressortir que, si la Fête Nationale que nous venions de célébrer le 8 mai était particulièrement française, celle qui nous réunissait le 14 juillet débordait les frontières de notre pays, en raison de l'influence considérable que l'idée sublime qui avait fini par se faire jour en 1789, avait exercée si heureusement sur le monde entier. Je n'ai pas manqué, à cette occasion, de rappeler que, comme elle l'avait fait à de si nombreuses époques de son histoire, la France avait ainsi réalisé l'une des plus grandes conquêtes morales de l'humanité dont, fraternellement, elle avait voulu que les autres nations partageassent le bénéfice avec elle ${ }^{61}$.

60) Ann-Laura Stoler, 2005 « Genre et moralité dans la construction impériale de la race », Actuel Marx 2005/2 ( $\left.\mathrm{n}^{\circ} 38\right)$, p. 83 .

61) Lettre du gouverneur des Etablissements français d'Océanie au Ministre des Colonies, du 20 juillet 1921 (Archives nationales d'Outre-mer, Aix-en-Provence, Fonds ministériels, Océanie, boîte 104). 
Cette légitimation de la «mission civilisatrice» s'accompagne d'un discours égalitaire entre les sexes : la France républicaine ne peut accepter des coutumes d'asservissement des femmes. Les missionnaires protestants de la LMS et de la SMEP ont également mis en avant la promotion de l'égalité des sexes pour justifier leurs actions d'évangélisation. Quel est le point de vue d'une femme missionnaire sur cette «mission civilisatrice»? Comment envisage-t-elle la place de la mission protestante dans cette colonie française?

Les propos d'Anne-Pauline Gilbert sur la colonisation évoluent au fil des pages. Au départ, elle évoque assez fréquemment « la mère patrie » notamment lors des préparatifs de son voyage en Suisse et en France et lors du premier culte au temple de Paofai à Papeete, au cours duquel elle se présente en ces termes :

On nous a demandé de parler [...] Je dis les salutations de l'église de Mulhouse à celle de Tahiti et la joie qu'elle a témoignée de ce qu'une Mulhousoise soit ici en mission. J'évoque aussi notre commune patrie française et les soldats tahitiens morts pour elle et j'ai le sentiment que cette dernière note «porte» dans l'auditoire (p. 62).

La «mère patrie » n'est plus évoquée sauf lors des fêtes du 14 juillet où elle compare le chant de La Marseillaise à un hymne de prière (p. 80) et les propos sur la colonisation s'infléchissent. Alors qu'à son arrivée, elle écrivait à propos de Tahiti : «l'île s'étant donnée à la France de son plein gré » (p. 64), elle remet en doute cette affirmation un an plus tard et s'interroge à demimot sur le bien fondé de la politique coloniale lorsqu'elle rencontre le fils de la reine Pomare :

A cette inauguration [de l'école pastorale de Hermon] assiste un personnage que je n'avais encore jamais rencontré : le prince Hinoï Pomaré, l'héritier du trône disparu. De ma vie, je n'ai vu un pareil géant ! [...] J'éprouve comme une gêne à la pensée que, sans nous les Français, ce peuple serait maître chez lui et cet homme un roi. Notre civilisation de blancs compense-t-elle leur indépendance ? Tahiti s'est «donné »..., évidemment (p. 145).

Cette remise en cause implicite des bienfaits de la colonisation française s'inscrit dans une réflexion plus générale sur la disparition de la «race» polynésienne qui inquiète aussi bien les autorités politiques que religieuses et qui sert de trame narrative à de nombreux romans. 
La chute démographique qui touche les îles de Polynésie est attestée tout au long du XIXe siècle : la population estimée à 32400 en 1874 n'est plus que de 22000 en 1892. La situation s'améliore à partir de 1920, passant de 30000 à 50000 personnes en 20 ans ${ }^{62}$.

Gustave Julien, gouverneur des Etablissements français d'Océanie de 1915 à 1919, établit en 1921 un rapport intitulé L'agonie d'une race. - Que faire ?63. Après avoir rappelé les surestimations des premiers circumnavigateurs et les nombreux facteurs de cette dépopulation (alcool, guerres, maladies importées, évangélisation et transformations trop rapides des mœurs et coutumes), le gouverneur Julien constate que « La race est sans résistance, sans réaction contre les agents extérieurs de sa ruine. Les purs indigènes tombent comme mouches, les métis résistent mieux » ${ }^{64}$. Face à ce constat, il prône, outre l'interdiction de l'alcoolisme et une immigration sélectionnée, le métissage pour enrayer la disparition de la race polynésienne :

[...] si nous voulons faire œuvre utile, enrayer la disparition trop rapide des survivants d'un beau rameau humain, envoyons en Océanie, à défaut de colons, des soldats de race blanche dont beaucoup se laisseront séduire par le charme des vahine, autant que par le calme idyllique d'une vie exempte de luttes et de trépidations [...] En envoyant, si la chose est possible, le plus d'éléments français que l'on pourra et, tout de suite, une garnison de bons soldats métropolitains, bien triés sur le volet, capables, s'ils y fondent des foyers, d'infuser un sang vigoureux et sain dans ce milieu où les rastaquouères exotiques tendent à prendre le dessus et à nous narguer chez nous ${ }^{65}$.

Les missionnaires français ne prônent pas une telle politique systématique du métissage ${ }^{66}$; ils rejoignent néanmoins le point de vue des autorités

62) Guy Guennou, François Merceron, Michel Lextreyt et Pierre-Yves Toulellan, Terres et civilisations polynésiennes, Paris, Editions Nathan, 1987, pp. 122-123 et p. 158.

63) «Colonies et marine» de mars-avril 1921, DEFAP.

64) Gustave Julien, L'agonie d'une race - Que faire?, 1921, p. 207.

65) Gustave Julien, L'agonie d'une race - Que faire?, 1921, pp. 207-209.

66) Aux Marquises, le gouverneur Julien recommande le métissage avec des «immigrants chinois ». Plusieurs missionnaires protestants considèrent quant à eux que les Chinois sont une menace pour les terres des Polynésiens (lettre de Maurice Leenhardt, Journal de la SMEP, 1926, p. 332). Paul-Louis Vernier, missionnaire aux Marquises, estime qu'un métissage entre Tahitiens et Marquisiens devrait sauver « la race marquisienne » (Journal de la SMEP, 1920, pp. 69-70). 
coloniales dans leur critique de la présence de «petits blancs » qui ont une influence néfaste sur les Polynésiens. Les missionnaires en viennent ainsi à distinguer une « bonne » civilisation - à la fois occidentale et chrétienne et une «mauvaise» civilisation - représentée par les touristes, les Européens de passage, les fonctionnaires et marins qui ne donneraient pas le bon exemple. Les effets néfastes de la civilisation (les bars et cinémas de Papeete, le matérialisme) qui iraient de pair avec un moindre sentiment religieux sont régulièrement mentionnés dans les comptes rendus missionnaires.

Le point de vue d'Anne-Pauline Gilbert est sensiblement différent. Elle n'évoque jamais la présence d'hommes blancs (non protestants) à Tahiti ; elle refuse le métissage ou en tout cas se sent mal à l'aise face aux femmes métisses même si dans le même temps elle éprouve de grandes difficultés à catégoriser les personnes :

Plus nos enfants, nos jeunes filles, se rapprochent du type tahitien pur, et plus elles me sont proches à moi, il me semble. C'est les «demies» qui si souvent me sont étrangères. Mes sentiments changeront-ils peu à peu à ce sujet? je suis dans un monde si peu stable et mes impressions sont si curieusement à fleur de peau en toutes choses... (p. 136).

Dans ce contexte, la mission de l'Eglise protestante est moins à ses yeux une mission civilisatrice - Anne-Pauline Gilbert regrette à plusieurs reprises une européanisation des comportements - qu'une mission réparatrice :

On n'a pas été sans remarquer que dans ce journal de missionnaire, la note religieuse manque. C'est que je ne la saisis pas de mes oreilles d'étrangère, de nouvelle. Pourtant, elle résonne, on me l'assure. En tout cas, il faut que l'œuvre missionnaire soit. Elle est une œuvre de justice et de réparation : partout où le blanc a passé, il y a à réparer. Ici, il y a de plus, une race à sauver de la disparition, de l'anéantissement. Qui pourrait le faire si ce n'est ceux qui viennent au nom de Dieu? (p. 115)

En conclusion de son journal le recentrement autour d'un engagement proprement religieux réapparaît avec force :

Si j'ai pu encourager quelque jeune personne à choisir la carrière missionnaire, j'en serai heureuse. Mais je le répète, il faut à ce métier difficile une sérieuse préparation. Et surtout qu'on ne parte plus sans savoir un peu la langue du pays où l'on se rend [...] Qu'on ne vienne pas du seul point de vue colonial! il faut, sans aucune austérité, une 
solide base religieuse, qui donne l'amour pour tout supporter. Et beaucoup de gaieté! (p. 156).

Pour mieux comprendre le point de vue d'Anne-Pauline Gilbert - qui éprouve une certaine nostalgie face à la disparition de la culture polynésienne - il est nécessaire outre les écrits missionnaires de tenir compte de l'abondante littérature sur l'Océanie qui s'est développée à partir de la fin du XIX ${ }^{\mathrm{e}}$ siècle et a nourri les désirs de voyage. Même si elle n'évoque pas précisément les motivations qui l'ont conduite à partir à Tahiti, la littérature a très certainement joué un rôle important: Anne-Pauline Gilbert cite notamment Jules Verne, le navigateur Alain Gerbault et Pierre Loti, dont le roman Le mariage de Loti publié en 1880, a rencontré un grand succès. Dans la littérature, les hommes tahitiens ne tiennent pas un rôle important, la majorité des romans privilégiant l'aventure entre un homme européen et une vahine tahitienne ${ }^{67}$. En arrière-plan, les hommes tahitiens symbolisent une civilisation ancienne en voie de disparition sous l'effet conjugué de la colonisation et de l'évangélisation ${ }^{68}$. C'est cette nostalgie d'une civilisation polynésienne en voie de disparition qui transparaît à plusieurs reprises dans le journal d'Anne-Pauline Gilbert et qui permet de mieux comprendre toutes les difficultés liées à la mise en cohérence de l'engagement missionnaire.

\section{De l'impossible voyage à l'engagement distancié}

Le journal d'Anne-Pauline Gilbert démarre avec le récit du voyage en bateau et prend la forme d'un journal de bord tenu quotidiennement. Ce voyage - dont le récit occupe un tiers du journal - constitue une expérience extraordinaire, un véritable rite de passage, ce qui justifie sa narration. A son arrivée à Tahiti, Anne-Pauline Gilbert évoque ainsi l'impression d'une «nouvelle naissance ${ }^{69}$ qui se traduit, dans les semaines suivantes, par une (re)découverte de soi et de sa foi protestante :

\footnotetext{
67) Margueron, 1989, pp. 243-244.

68) Ibid.

69) Le jour d'accostage du bateau, Anne-Pauline Gilbert écrit ainsi : «Nous disparaissons sous les fleurs que les élèves entassent sur nos genoux, et j'ai soudain la réconfortante vision
} 
Je me forme à la pédagogie tropicale et insulaire. «Ici », dit Melle P. « on devient tout à fait bon, ou foncièrement mauvais». Les élèves essayent de m’aiguiller sur la voie la plus conforme à mon tempérament. « Tamata = essayer », c'est leur devise [...] La Bible est ici le livre, et l'indigène, si près de la nature, de l'humanité primitive, s'intéresse vivement aux récits de l'ancien testament. Ces récits prennent d'ailleurs pour moimême, un relief, une vie étonnante depuis que je suis ici (p. 73).

Mais une fois passées les premières semaines, les excursions hors de Papeete deviennent rares et les visites dans les autres îles de Polynésie inexistantes. Plusieurs raisons permettent d'expliquer les difficultés à poursuivre, à terre, le voyage : l'interdiction pour les femmes missionnaires de voyager seules, la surcharge de travail et l'absence de transport régulier. Les femmes occidentales ne doivent pas voyager seules, notamment pour effectuer les grands voyages maritimes, Anne-Pauline Gilbert est ainsi accompagnée d'Emilie Banzet lors de sa première traversée ${ }^{70}$. Mais cette règle n'apparaît pas, en définitive, très contraignante puisqu'Anne-Pauline Gilbert peut prendre le truck (petit bus polynésien) pour rentre visite aux infirmières d'Orofara, à une heure de route; elle effectue également avec une autre collègue un tour de lîle. Une femme missionnaire peut également être accompagnée d'une «aide indigène» ou des jeunes filles de l'internat ${ }^{71}$.

de mon enterrement» (p. 58). «L'impression n'est nullement celle du «déjà vu», déjà «vécu» cher aux «transmigrationnistes». Non, je vis du tout nouveau, je vais vers l'inconnu, vers les luttes qui me demanderont toute entière. Le mieux sera donc de se comporter, surtout vis-à-vis de soi-même, comme si on était fait à cela depuis longtemps, depuis toujours...» (p. 57).

70) Cette règle est officiellement abrogée en 1936 pour les deux infirmières envoyées par la Société afin d'assurer en permanence la présence d'une infirmière sur place pendant que sa collègue rentre en congé régulier en Métropole (courrier du 17 août 1936 adressé à Messieurs les membres du comité des Missions).

71) La longue description d'une sortie avec des jeunes filles de l'internat et quelques anciennes élèves à la cascade de la Fautaua illustre cette relative liberté de déplacement associée à un incorporation distanciée des normes de genre. «Un pique nique à la Tahitienne doit être un entreprise formidable? Nous qui n'avons pas de temps à perdre en luxueux préparatifs, nous n'emportons pas la plus belle vaisselle possible, ni l'argenterie de choix [...] Ni café, ni champagne, on boira l'eau des ruisseaux qui jasent sous les immenses fougères et notre vaisselle plate sera les feuilles luisantes de ces fougères. Chacune porte son sac et son paquet; il faut une robe de rechange, des sandales peut-être pour la baignade. Les enfants préfèrent être pieds-nus ». (p. 97). 
C'est davantage la surcharge de travail et la fatigue des transports qui ne permettent pas, comme Anne-Pauline Gilbert l'aurait souhaité, de découvrir pleinement les modes de vie et la culture polynésiennes. Et lorsque l'occasion se présente, les descriptions laissent deviner une certaine déception. A la recherche de l'exotisme, Anne-Pauline Gilbert découvre une société polynésienne profondément évangélisée et « européanisée »; à la recherche d'un idéal masculin «authentique», elle ne peut pas nouer des contacts avec des hommes tahitiens, en raison de son statut de femme missionnaire contrainte au célibat. Son journal lui permet néanmoins de laisser libre cours à ses rêveries sentimentales ${ }^{72}$.

Le lecteur entrevoit en fait dès les premières pages cet «impossible voyage ${ }^{73}$ lorsque après avoir évoqué le mal de mer Anne-Pauline Gilbert se demande en découvrant les Antilles si elle n'aurait pas aussi «le mal de terre ». Mais c'est surtout à travers la description des paysages à Tahiti qu'elle évoque cet impossible voyage qui prend la forme d'un léger malêtre dû au décalage entre l'expérience vécue et l'idéal nourri d'abondantes lectures :

Je quitte à regret l'asile de paix [la demeure des infirmières de la léproserie où elle est venue se reposer] n'emportant qu'une impression désagréable - il en faut une apparemment - ma brouille avec les cocotiers [...] les cinq ou six longs flandrins qui sont de garde devant la case d'Orofara se sont amusés à me faire d'inoubliables grimaces se détachant sur l'horizon infini à l'heure des contemplations sentimentales» (p. 122). « «Un paysage est un état d'âme», a dit Amiel. Parce que mon âme est toujours absente, rien de toute cette beauté ne peut y créer de la joie, de la vie. Mais, c'est vrai : Tahiti est beau (p. 146).

72) Lors du défilé du 14 juillet Anne-Pauline Gilbert écrit ainsi : «un groupe d'hommes a particulièrement belle allure : le maillot bleu clair moule des torses impeccables, la ceinture-jupe, faite de longues franges en minces lanières d'écorce de «burao » [pûrau, nom d'un arbre] tombe jusqu'au genoux sur les jambes nues. D'autres groupes ont conservé et c'est regrettable, le long pantalon blanc sous la jupe frangée » (p. 83). Lors d'un repas officiel en l'honneur de Monsieur Allégret, elle regrette de s'être endormie : « Ni les beaux récits de Monsieur ALLEGRET qui nous parlait de ses souvenirs d'Afrique, ni le discours puissamment modulé du robuste chef de Papenoo à côté de qui j'étais assise et que j'évoquais avec la ferveur du désespoir en pareu [paréo] et plumes sur la tête (il était en noir et blanc) ne purent me tirer de mon invincible torpeur [...]. Parlotes, types authentiques, chefs et chanteurs, yeux ardents et chevelures admirables tout se noie dans le même néant» (p. 109). 73) Marc Augé, L'impossible voyage. Le tourisme et ses images, Paris, Payot \& Rivages, 1997. 
Il faut lire la correspondance avec le directeur de la mission pour comprendre le sens de cette «brouille avec les cocotiers», cette note dissonante dans le paysage qui est une allusion à son incapacité à s'engager corps et âme dans l'activité missionnaire et la réalité polynésienne. En mars-avril 1928, deux ans après son arrivée à Tahiti, Anne-Pauline Gilbert écrit au directeur de la SMEP :

Le temps que je pensais pouvoir consacrer à l'œuvre de Tahiti, trois ans, expirant en mai 1929, je serais heureuse de savoir que des démarches sont faites dès à présent, par le Comité de Paris, afin de me trouver une remplaçante pour la rentrée d'automne de la dite année ${ }^{74}[\ldots]$ L'Ecole va en augmentant, il lui faut des forces neuves, de grandes forces. Je n'ai jamais regretté d'être venue à Tahiti, mais je n'ai pas été «prise » par l'œuvre, en aucune façon, à aucun moment. Je ne pourrais donc y rester pendant de longues années. D'ailleurs, j'ai passé l'âge où l'on s'attend à grandir en force, j'ai peutêtre lieu de m'attendre au contraire ${ }^{75}$.

Ce « voyage impossible» se transforme en engagement distancié. La position de bénévole d'Anne-Pauline Gilbert lui permet d'entretenir un regard critique sur la mission protestante à Tahiti ainsi que sur son propre engagement missionnaire. Anne-Pauline Gilbert n'hésite pas à écrire qu'elle chante faux ou qu'elle s'endort pendant les cultes, donnant à voir une image profondément différente de celle, idéalisée, des missionnaires faisant entièrement «corps » avec l'œuvre missionnaire. Alors que la discipline de l'Eglise est particulièrement stricte, Anne-Pauline Gilbert écrit à plusieurs reprises qu'elle rencontre des difficultés à faire classe dans le calme : «Un jour, à travers les cours, l'on m'envoie un émissaire «Si Mlle Gilbert pouvait faire taire un peu les petits, on ne s'entend pas bien dans les autres classes » (p. 135); ces difficultés de discipline sont directement liées à son incapacité assumée à punir les enfants : « je punis à contre-cœur car je ne crois pas à l'efficacité des punitions, et toujours à froid. Qu'attendre d'ailleurs, dans cet ordre d'«idées » d'une éducatrice qui est obligée de se stimuler elle-même par un «fâche-toi maintenant, il en est temps » (p. 84-85).

74) Lettre du 5 mars 1928 de Anne-Pauline Gilbert (Papeete) adressée à Monsieur Allégret.

75) Lettre personnelle d'A.-P. Gilbert du 5 mars 1928, adressée à Monsieur Allégret. 
Que cache cette absence de discipline revendiquée ? Une certaine complicité avec les élèves, mais aussi une recherche de liberté qui a du mal à se concrétiser dans le cadre missionnaire ${ }^{76}$. Mais cette indiscipline, toute relative, ne remet en cause ni le travail effectué ni la fréquentation, en hausse, de l'école maternelle :

Sous la direction éclairée et affectueuse de Mlle Gilbert, l'Ecole maternelle n'a fait que s'accroître. Le total le plus élevé des élèves a été de cent vingt-deux. Mlle Gilbert préside le culte, donne des leçons de solfège et de chant, de récitation française, sans oublier contes et récits mimés, tandis que ses aides «font de leur mieux auprès des a.b.c.istes, leur enseignant à tenir le crayon tant convoité » 77 .

Anne-Pauline Gilbert quitte Tahiti en août 1931, après y avoir passé plus de 5 ans, et fait part, en mars 1933, de son désir de revenir en Polynésie, en faisant valoir le soutien des missionnaires de Tahiti :

Puis-je vous annoncer que je serais prête à retourner à Tahiti en juin prochain, à mes frais et à tire d'aide au pair, avec votre autorisation naturellement?

Ayant été avertie ici que dans vos Bureaux [à Paris] je suis jugée insuffisante à mon poste, j'ai insisté auprès de notre Conférence Missionnaire [à Tahiti] pour avoir et un certificat sur mon activité passée dans l'Ecole et un appel ferme pour mon retour éventuel à ce poste. Les deux documents sont rédigés avec tant de chaleur que je ne puis plus croire que mon retour là-bas serait une erreur. [...] $]^{78}$ Et je veux accepter ce nouveau départ comme voulu de Dieu, car personnellement je n'ai pas plus d'enthousiasme qu'en 1926 et très peu de courage ${ }^{79}$.

76) La similitude de position entre ceux qui n'ont pas encore lâge de se conformer strictement aux normes comportementales et ceux qui estiment avoir passé l'âge permet ainsi à Anne-Pauline Gilbert de concilier son aspiration à une relative liberté comportementale et ses obligations missionnaires: «...tous les matins, dans l'une des grandes cours, je fais la gymnastique avec mon bataillon de va-nu-pieds. C'est ça qu'il faut voir !!! Personne de nous ne sachant quel est son pied gauche ou sa jambe droite, car moi je l'ai oublié dans l'ardeur du combat, nous brandissons dans la direction qui nous plait, le membre qu'il nous plait. Ou bien nous ne brandissons rien du tout, nous contentant de rire de la nouvelle maîtresse qui se démène si drôlement. Et l'alignement s'en ressent !!! » (p. 134).

77) Journal de la SMEP, mars 1931, Rapport de la mission de Tahiti, exercice 1929-1930, p. 204.

78) Courrier du 3 mars 1933 d'A.-P. Gilbert (Mulhouse) adressé à Messieurs Allégret et Couve.

79) Lettre du 23 mars 1933 d'Anne-Pauline Gilbert (Mulhouse) adressée à Monsieur le directeur. 
Anne-Pauline Gilbert repart à Tahiti en 1933 ; elle rentre définitivement en Métropole en 1950, elle est alors âgée de 73 ans.

Conclusion : (In)égalité de sexe, (in)égalité de « race » : projets missionnaires et réalités polynésiennes

Le départ d'Anne-Pauline Gilbert pour Tahiti en 1926 s'inscrit dans une dynamique, initiée dès les années 1880 , de féminisation des organisations missionnaires protestantes liée, pour les femmes célibataires, à une professionnalisation du travail missionnaire centré sur les métiers d'institutrice puis d'infirmière. Cette professionnalisation autorisa une autonomie relative par rapport à l'organisation missionnaire, renforcée, dans le cas d'Anne-Pauline Gilbert, par le caractère bénévole de son engagement : elle repart ainsi à Tahiti en 1933 contre l'avis de certaines personnes de la SMEP à Paris. Anne-Pauline Gilbert illustre également une diversification des parcours et des motivations des missionnaires puisque son engagement individuel s'éloigne du programme missionnaire officiel de conversion et de «mission civilisatrice».

La lecture du journal d'Anne-Pauline Gilbert permet de mettre en évidence, à côté de l'évangélisation et des activités ecclésiastiques, l'importance de l'instruction au sein de la Mission protestante en Polynésie, notamment auprès des jeunes filles polynésiennes. Mais la finalité ultime de cette instruction est l'objet d'interprétations différentes, les uns estimant que le mariage - et avec lui la moralisation des familles polynésiennes censée sauver la «race» polynésienne - est l'objectif auquel ces jeunes filles doivent se préparer, les autres (notamment les institutrices missionnaires) estimant au contraire qu'un emploi salarié dans les écoles protestantes ou laïques constitue une ambition professionnelle légitime pour les jeunes filles diplômées. Alors que la Mission protestante a fait de l'apprentissage de la domesticité et de l'inculcation des rôles d'épouse et de mère un des objectifs majeurs de son action auprès des Polynésiennes, les institutrices célibataires de l'école des jeunes filles sont une exception remarquable à ce modèle idéalisé de la famille chrétienne et un exemple possible à suivre pour les élèves. Les champs missionnaires ont d'ailleurs constitué une opportunité, pour les femmes occidentales qui ne voulaient pas être cantonnées à la sphère domestique, d'exercer des 
activités professionnelles. Les hommes et les femmes missionnaires n'ont donc pas toujours la même compréhension de l'égalité des sexes et des modalités de promotion de cette égalité parmi les Polynésiens. Les débats sur l'autorisation du divorce montrent ainsi comment la promotion de l'égalité des sexes auprès des Polynésiens est un argument rhétorique qui permet de penser le maintien de l'inégalité des « races » et de justifier une politique disciplinaire inégalitaire en matière d'adultère.

Analysant le genre et la moralité dans la construction impériale de la «race», Ann-Laura Stoler rappelle qu'au début du XXe siècle, les communautés européennes dans les colonies n'apparaissent déjà plus comme des communautés homogènes, les divisions entre missionnaires et administrateurs politiques, entre «petits blancs» et riches entrepreneurs reflétant la concurrence entre projets politiques et économiques divergents ${ }^{80}$. C'est dans ce contexte historique où « les marqueurs de l'identité européenne et les critères d'appartenance à la communauté n'apparaissent plus comme fixes, mais se révèlent un terrain manifestement plus fluide, plus perméable, et historiquement disputé » que les relations entre les sexes sont repensées ${ }^{81}$.

A Tahiti, le métissage et l'arrivée de «petits blancs» rendant la pertinence des catégories de «race» et de classes sociales problématique, les missionnaires protestants mettent en avant l'importance de la moralité comme critère de catégorisation et de distinction sociale, mais ce critère est lui-même l'objet de redéfinitions et de reformulations ${ }^{82}$. Pour les missionnaires protestants représentant la Conférence de Tahiti la moralité est indissociable des questions sexuelles (l'interdiction de relations sexuelles en dehors des liens du mariage); pour Anne-Pauline Gilbert l'idéal de

80) Ann-Laura Stoler, 2005 « Genre et moralité dans la construction impériale de la race », Actuel Marx 2005/2 ( $\left.\mathrm{n}^{\circ} 38\right)$, pp. 78-79.

81) Ibidem. Et c'est dans l'entre-deux-guerres que les épouses européennes voient leur rôle de gardiennes des normes occidentales renforcé.

82) Après un séjour en Polynésie en 1926, le missionnaire Maurice Leenhardt décrit ainsi les « demi-blancs » en termes péjoratifs tout en concluant: «Loin de nous la pensée de prétendre que tous les demi-blancs soient ainsi, puisque l'Eglise et le pastorat en compte un très grand nombre, mais leur foule crée près du vieux roc tahitien, un fond mouvant qui devient assez considérable pour constituer le fond même du peuple tahitien actuel. Et l'Evangile seul peut fixer ce fond mouvant» (JSMEP, 1926 p. 333). 
moralité - incarné par les deux infirmières de la léproserie - renvoie davantage aux notions de dévouement, de courage et d'abnégation.

Si le Journal d'Anne-Pauline Gilbert n'insiste pas sur les divergences d'opinion au sein du «corps» missionnaire, il permet en revanche, par contre-point, de mieux comprendre l'imbrication des hiérarchies de sexe et de « race » constitutives des dynamiques missionnaires pendant l'entredeux-guerres. Alors que Charles Vernier exprime, dans ses comptes rendus publiés dans le Journal de la SMEP, une vision très hiérarchisée de la mission protestante à Tahiti, Anne-Pauline Gilbert revendique comme idéal l'égalité des sexes et l'égalité des « races » 83 .

L'hypothèse peut être faite que c'est sa position sociale dominée en tant que femme bénévole au sein du champ missionnaire qui lui fait prendre conscience des autres formes d'inégalité et notamment des inégalités de « race ». La lecture de son journal suggère une interprétation complémentaire: l'égalité des races qu'elle mentionne dès l'escale aux Antilles - «le principe tant chéri de l'égalité des races » (p. 20) - est en fait un préalable à son engagement missionnaire. Derrière la remise en cause de certains stéréotypes véhiculés sur les Polynésiens, le lecteur décèle une sensibilité particulière: celle d'une femme qui a fait l'expérience des assignations de genre contradictoires. En d'autres termes, Anne-Pauline Gilbert est d'autant plus sensible à la rhétorique missionnaire de la superficialité qui s'applique aux Polynésiens qu'elle fait écho à un mécanisme similaire de disqualification des femmes occidentales qui lorsqu'elles se conforment aux normes de féminité sont aussi susceptibles d'en faire trop. Consciente que l'équilibre est difficile à tenir entre une injonction à la féminité et une féminité superficielle, Anne-Pauline Gilbert ne s'approprie donc pas la critique d'un protestantisme polynésien superficiel (même si son journal n'est pas exempt d'autres stéréotypes) alors que cette critique est un des traits structurants du discours missionnaire de légitimation du maintien

\footnotetext{
83) Le lien entre ces deux idéaux égalitaires est particulièrement fort lors de la description du défilé du 14 juillet auquel participent les enfants scolarisés :

«Défilé de la toute petite garnison de Tahiti défilé des marins de notre aviso de guerre, le C[assionée], en rade ici depuis quelques semaines, défilé des Sociétés de sport, des Ecoles. Le gouverneur aurait été particulièrement touché par l'entrain des fillettes qui, virilement emboîtaient le pas derrière l'élément masculin. Et nos enfants trouvent que les soldats tahitiens portent le fusil même mieux que les marins du C...! (p. 8o).
} 
des hiérarchies raciales. Anne-Pauline Gilbert est particulièrement sensible aux critiques du caractère excessif des marques de féminité: la coquetterie, la sienne, est à bannir ; elle tente donc d'élaborer auprès de ses élèves des normes de féminité a minima qui intègrent cette critique en allant vers la «simplicité». Et c'est aussi ce qu'elle cherche en venant en Polynésie : «la simplicité », l'authenticité, la découverte de la nature et de la culture polynésiennes.

Mes travaux de thèse, à partir de l'analyse de la littérature missionnaire classique où s'affirme notamment l'idée d'un «zèle» féminin à encadrer, ont montré que les Polynésiennes avaient pu s'approprier les discours missionnaires et mettre en place des lieux de sociabilité féminine, illustrant une intégration partielle et distanciée des normes religieuses et de genre ${ }^{84}$. Le Journal d'Anne-Pauline Gilbert comparé à celui de la SMEP offre un déplacement du regard heuristique, qui permet de découvrir une relative hétérogénéité des missionnaires à Tahiti, selon leur âge, leur sexe, leurs fonctions, leurs motivations et leurs parcours personnels. Or, les possibilités de réappropriations et d'adaptions des normes de genre ont pu apparaître d'autant plus importantes que « le corps missionnaire » ne présentait pas un programme homogène en la matière. En d'autres termes les marges d'autonomie et les marges de liberté des jeunes filles polynésiennes ont pu être d'autant plus importantes que différents modèles légitimes de féminité leur étaient proposés, des institutrices célibataires aux épouses de missionnaires.

84) Malogne-Fer, 2007, pp. 43-47. 